\title{
Molecular Alterations in the Cerebellum of Sporadic Creutzfeldt-Jakob Disease Subtypes with DJ-1 as a Key Regulator of Oxidative Stress
}

\author{
Waqas Tahir $^{1}$ • Saima Zafar ${ }^{1} \cdot$ Franc Llorens $^{1} \cdot$ Amandeep Singh Arora ${ }^{1}$. \\ Katrin Thüne $^{1}$ - Matthias Schmitz ${ }^{1}$ - Nadine Gotzmann ${ }^{1}$ • Niels Kruse ${ }^{2}$ • \\ Brit Mollenhauer $^{2}$ • Juan Maria Torres ${ }^{3}$. Olivier Andréoletti ${ }^{4} \cdot$ Isidre Ferrer $^{5,6}$. \\ Inga Zerr ${ }^{1}$
}

Received: 2 September 2016 / Accepted: 8 November 2016

(C) Springer Science+Business Media New York 2016

\begin{abstract}
Cerebellar damage and granular and Purkinje cell loss in sporadic Creutzfeldt-Jakob disease (sCJD) highlight a critical involvement of the cerebellum during symptomatic progression of the disease. In this project, global proteomic alterations in the cerebellum of brain from the two most prevalent subtypes (MM1 and VV2) of sCJD were studied. Twodimensional gel electrophoresis (2DE) coupled mass spectrometric identification revealed 40 proteins in MM1 and 43 proteins in VV2 subtype to be differentially expressed. Of those, 12 proteins showed common differential expression in their expression between two subtypes. Differentially expressed proteins mainly belonged to (i) cell cycle, gene expression and cell death; (ii) cellular stress response/ oxidative stress (OS) and (iii) signal transduction and synaptic functions, related molecular functions. We verified 10 differentially expressed proteins at transcriptional and translational level as well. Interestingly, protein deglycase DJ-1 (an antioxidative protein) showed an increase in its messenger RNA
\end{abstract}

Electronic supplementary material The online version of this article (doi:10.1007/s12035-016-0294-4) contains supplementary material, which is available to authorized users.

Saima Zafar

sz_awaan@yahoo.com

1 Department of Neurology, University Medical Center Goettingen (UMG) and German Center for Neurodegenerative Diseases (DZNE) Goettingen, Robert-Koch-Str., 40, 37075 Goettingen, Germany

2 Institute of Neuropathology, University Medical Center Goettingen (UMG), Robert-Koch-Str. 40, 37075

Goettingen, Germany
(mRNA) expression in both MM1 and VV2 subtypes but protein expression only in VV2 subtype in cerebellum of sCJD patients. Nuclear translocalization of DJ-1 confirmed its expressional alteration due to OS in SCJD. Downstream experiments showed the activation of nuclear factor erythroid-2 related factor 2 (Nrf2)/antioxidative response element (ARE) pathway. DJ-1 protein concentration was significantly increased during the clinical phase in cerebrospinal fluid of SCJD patients and also at presymptomatic and symptomatic stages in cerebellum of humanized PrP transgenic mice inoculated with SCJD (MM1 and VV2) brain. These results suggest the implication of oxidative stress during the pathophysiology of SCJD.

Keywords Sporadic Creutzfeldt-Jakob disease . Two-dimensional gel electrophoresis · Mass spectrometry . Parkinson disease protein 7/protein deglycase DJ-1 . Antioxidative response elements
Instituto Nacional de Investigación y Tecnología Agraria y Alimentaria, Carretera de Algete a El Casar Km. 8,1 S/N, 28130 Valdeolmos, Madrid, Spain

4 Institut National de la Recherche Agronomique/Ecole Nationale Vétérinaire, Toulouse, France

5 Institute of Neuropathology, Hospitalet de Llobregat, IDIBELL-University Hospital Bellvitge, University of Barcelona, Barcelona, Spain

6 Network Center for Biomedical Research of Neurodegenerative Diseases (CIBERNED), Ministry of Health, Institute Carlos III, Madrid, Spain 


$\begin{array}{ll}\text { Abbreviations } \\ \text { sCJD } & \text { Sporadic Creutzfeldt-Jakob disease } \\ \mathrm{PrP}^{\mathrm{C}} & \text { Cellular prion protein } \\ \mathrm{PrP}^{\mathrm{Sc}} & \text { Scrapie form of prion proteins } \\ \text { 2DE } & \text { Two-dimensional gel electrophoresis } \\ \mathrm{OS} & \text { Oxidative stress } \\ \mathrm{CSF} & \text { Cerebrospinal fluid }\end{array}$

\section{Introduction}

Prion diseases are transmissible spongiform encephalopathies [1] which can be sporadic, inherited or acquired in their origin [2]. Sporadic prion diseases account for $85 \%$ of all human prion diseases [3], and SCJD comprises of more than 85$90 \%$ of all sporadic prion diseases [3]. Heterogeneity of clinic-histopathological features as well as classification of sCJD is largely dependent on genotype at codon 129 (M/M, $\mathrm{M} / \mathrm{V}$ or $\mathrm{V} / \mathrm{V}$ ) of $P R N P$ gene (encoding prion protein) and type 1 or 2 of scrapie form of prion protein $\left(\mathrm{PrP}^{\mathrm{Sc}}\right)$ [2]. Homozygosity at codon of 129 of PRNP gene for methionine with type $1 \mathrm{PrP}^{\mathrm{Sc}}$ (MM1 subtype) and valine with type $2 \mathrm{PrP}^{\mathrm{Sc}}$ (VV2 subtype) accounts for the two most prevalent subtypes (67 and 15\% respectively) of sCJD [2, 4, 5]. Differential selection of the brain regions for heterogeneous pathological features by genotype at codon 129 of PRNP demands for subtype and brain region-specific targeted studies. The cerebellum presents pathological features like synaptic degeneration, loss of granular, molecular as well as Purkinje cell layers due to PrP deposition and gliosis in SCJD patients [6]. This can lead to dysregulation of important physiological functions of cerebellum-like voluntary movements of the body [7, 8] implicating its relation to clinical symptoms like myoclonus and pyramidal/extrapyramidal signs shown by sCJD patients [5]. Diverse pathological events occurring in the cerebellum during the pathophysiology of SCJD are still not completely explored. Identification and characterization of proteomic alterations of sCJD subtypes is important to understand the disease pathology and development of various clinicopathological phenotypes. Proteome-wide studies using high-throughput differential proteomic approaches can be helpful in mapping the whole proteomic regulations by identification of expressionally altered proteins, protein abundance, altered folds of their expression, post translational modifications and protein-protein interactions and identification of networks to understand the underlying cellular processes [9].

So, this study was planned with the objectives to explore the global proteomic alterations in the cerebellum of the two most prevalent SCJD (MM1 and VV2) subtypes by using twodimensional gel electrophoresis (2DE) and mass spectrometry, altered cellular activities, validation of identified altered proteins at transcriptional and translational level and alteration of a key player/pathway during the pathophysiology of disease. Proteomic findings in this study suggested a pronounced implication of oxidative stress (OS) during the pathophysiology of sCJD shown by broad spectrum of proteomic alterations associated with oxidative stress. Oxidative stress is one of the key features in the pathophysiology of prion diseases [10], but it is under privileged in SCJD. Expression of Parkinson's disease-associated protein DJ-1 was also identified to be altered in SCJD. Further investigations were made to unveil the expressional alteration of protein DJ-1 during the pathophysiology of disease and its downstream nuclear factor erythroid-2 related factor 2 (Nrf2)/antioxidative response element (ARE) pathway in SCJD. This study provides the first comprehensive analysis of differential proteomic alterations in the cerebellum SCJD (MM1 and VV2) subtypes. These results imply the role of oxidative stress during the pathophysiology of sCJD as shown by broad spectrum of regulations associated with OS.

\section{Materials and Methods}

\section{Sample Collection and Processing}

Cerebellum tissue samples from postmortem brains of pathologically confirmed SCJD patients were received from the Brain Bank of Institute of Neuropathology HUB-ICOIDIBELL and Clinic Hospital-IDIBAPS Biobank according to the Spanish legislation on the collection and transport of biological samples and also after the approval from local ethics committee. Postmortem interval (PMI) was between $1 \mathrm{~h}$ and $45 \mathrm{~min}$ to $24 \mathrm{~h}$ and $30 \mathrm{~min}$ which primarily does not affect the expression of proteins in the tissue [11]. Collection and processing of brain samples were performed as described previously $[12,13]$. Four samples (each from MM1 and VV2 subtypes along with their age-matched non-demented controls (NDC)) were used for proteomic study. Mean age of the samples from age-matched NDC, MM1 and VV2 subtypes along with their standard deviations were $63 \pm 12.19,70 \pm 8.83$ and $72 \pm 3.74$ respectively. All the brain samples used for this study were from female patients.

\section{Antibodies, Oligonucleotides, Kits and Commercial Buffers}

All primary antibodies, secondary antibodies, oligonucleotides, kits and commercial buffers used in this study are listed in the supplementary Tables 1,2,3 and 4 respectively.

\section{Sample Preparation, Proteinase K Digestion, SDS-PAGE and Western Blotting}

Tissue lysates $(10 \% w / v)$ of frozen cerebellum tissue samples were prepared by homogenization in lysis buffer $(7 \mathrm{M}$ urea, 
$2 \mathrm{M}$ thiourea, 4\% CHAPS, $20 \mu \mathrm{l} / \mathrm{ml}$ ampholytes, $10 \mathrm{mg} / \mathrm{ml}$ dithiothreitol (DTT), protease and phosphatase inhibitors). The protein concentrations in tissue lysates were determined by using Bradford assay (Bio-Rad). For proteinase K (PK) digestion, $200 \mu \mathrm{g}$ of proteins from cerebellum of sCJD brain $(10 \% \mathrm{w} / \mathrm{v})$ lysates (both MM1 and VV2 subtypes along with age-matched NDCs) were digested with $2.5 \mu \mathrm{g} / \mathrm{ml}$ of PK for $1 \mathrm{~h}$ at $37{ }^{\circ} \mathrm{C}$, and the enzymatic activity was stopped by heating the samples later at $65^{\circ} \mathrm{C}$ for $15 \mathrm{~min}$. Then, samples were boiled at $95^{\circ} \mathrm{C}$ for 5 min after mixing them with $4 \times$ RotiLoad (ROTH) as sample loading buffer. Samples were cooled down and were used further for sodium dodecyl sulphatepolyacrylamide gel electrophoresis (SDS-PAGE) and western blotting as described previously [14].

\section{Two-Dimensional Gel Electrophoresis, Visualization and Analysis of Protein Spots}

2DE is based on the separation of proteins in two dimensions: based on isoelectric focusing (IEF) in the first dimension and molecular weight with SDS-PAGE in the second dimension and was performed as described previously [15]. Protein spots on 2DE gels were visualized with silver stain [16] followed by scanning (CanoScan Scanner) for further analysis of protein spots. Differential expression of protein spots between different groups was analysed in terms of the intensity of each spot with the help of DECODON Delta2D software and calculated by using a cut-off value of 1.5 -fold change and $p$ value $<0.05$ in unpaired Student's $t$ test.

\section{Identification of Protein/Peptide Sequences by Mass Spectrometry}

Protein spots with altered expression were excised from silverstained 2DE gel and were further processed (destaining of silver stain, reduction of disulphide bonds, alkylation of free cysteines, trypsin digestion, peptide extraction and identification of sequence of peptides) for identification of sequence of proteins with Q Exactive hybrid quadrupole/orbitrap mass spectrometry as described [14]. After identification of proteins with mass spectrometry, stringent parameters of peptide count $(\geq 2)$, maximum peptide threshold $(95 \%)$ and minimum false discovery rate (FDR) $(0.01 \%)$ were further employed during the qualification of identified proteins to exclude any possibility of false positive identifiers in the proteomic data for further analysis.

\section{mRNA Expression of Identified Proteins for Validation}

sCJD samples including MM1 $(n=15)$ and VV2 $(n=10)$ subtype from the cerebellum part of brain tissue from pathologically confirmed that SCJD cases and their age-matched NDC ( $n=15)$ were used for analysing the messenger RNA
(mRNA) expressions of selected proteins for validation. Mean age of the samples from age-matched NDCs, MM1 and VV2 subtypes along with their standard deviations were $62.13 \pm 12.06,65.93 \pm 14.55$ and $63 \pm 12.09$ respectively.

\section{RNA Isolation, Purification, Reverse Transcription Reaction} and $q P C R$

Extraction and purification of RNA, reverse transcription and qPCR reactions were performed as described previously $[12,13]$. Briefly, extraction and purification of RNA were performed by using mirVana microRNA (miRNA) isolation kit (Ambion AM1560) according to the instructions of the manufacturer. The concentration of extracted RNA was measured at $260 / 280$ by using Nanodrop 2000 spectrophotometer (ThermoScientific, USA). The reverse transcription reaction was performed with the HighCapacity cDNA Archive kit (Applied Biosystems) according to the instructions of the manufacturer by using the C1000 Touch Thermal Cycler (Bio-Rad).

Duplicate reactions of each cDNA sample were used for performing PCR reaction in 384-well optical plates (Roche) by using 20× TaqMan Gene Expression Assays (Applied Biosystems) and $2 \times$ TaqMan Universal PCR Master Mix (Applied Biosystems). $\beta$-Glucuronidase (GUS $\beta$ ) was used as an internal control for normalization. PCR reaction was carried out in LightCycler 480 II (Roche) with the following settings: step $150{ }^{\circ} \mathrm{C}$ for $2 \mathrm{~min}$, step 2 denaturation at $95^{\circ} \mathrm{C}$ for $10 \mathrm{~min}$ followed by 40 cycles for annealing, step $395^{\circ} \mathrm{C}$ for $15 \mathrm{~s}$ and step $460^{\circ} \mathrm{C}$ for $1 \mathrm{~min}$. TaqMan PCR data were retrieved using the Sequence Detector Software (SDS version 2.1, Roche). Subsequently, cycle threshold (CT) data for each sample were analysed with the double delta CT $(\Delta \Delta \mathrm{CT})$ method.

\section{CJD MM1 and VV2 Transgenic Mice}

PrP knockout transgenic mice that expressed human cellular prion protein $\left(\mathrm{PrP}^{\mathrm{C}}\right)$ having either methionine (129MetTg340) or valine (129Val-Tg361) at codon 129 were used to develop humanized models of CJD as previously described [17-20]. Both 129Met-Tg340 and 129Val-Tg361 mouse lines were homozygous for human PRNP gene and showed similar brain expression levels of $\operatorname{PrP}^{\mathrm{C}}$ (around 4-fold the expression level in human brain) on a mouse PrP null background. These two mouse lines were used for developing MM and VV mice models of sCJD separately by intracranial inoculation of $10 \%$ $(w / v)$ homogenate from either sCJD MM1 or SCJD VV2. Collection of brain samples of the inoculated mice along with their age-matched healthy controls was done post inoculation at days 120, 160 and 183 for preclinical, clinical and late clinical stages for MM transgenic mice and at days 120, 160 
and 180 for early clinical, clinical and late clinical to terminal stages for VV transgenic mice respectively.

\section{Co-Immunofluorescence}

Formic acid-treated frozen tissue samples from the cerebellum region of brain from CJD (MM1) and age-matched NDC cases were paraffin embedded and fixed with paraformaldehyde. Slides with 4- $\mu$ m sections were washed with PBS, and antigenic site on tissue sections was exposed by permeabilization buffer (PBS $+0.2 \%$ Triton X-100) for $10 \mathrm{~min}$. Non-specific proteins were blocked by using $5 \%$ bovine serum albumin in PBS for $2 \mathrm{~h}$ followed by overnight incubation with anti-DJ-1 (1:100) primary antibody (pAb) diluted in 5\% serum with $\mathrm{PBS}$ for overnight at $4{ }^{\circ} \mathrm{C}$. Horseradish peroxidase (HRP)-labelled anti-goat secondary antibody was used for $2 \mathrm{~h}$, and nuclei were stained with DAPI $(0.1 \mu \mathrm{g} / \mathrm{ml}$ of PBS $)$ for $10 \mathrm{~min}$. Slides were visualized using a fluorescence microscope. Image J (WCIF plugin) software was used for analysing co-localization pattern of individual images.

\section{Electrochemiluminescence-Based Enzyme-Linked Immunosorbent Assay}

Electrochemiluminescence (ECL)-based ELISA was performed to analyse the expression of DJ-1 in cerebrospinal fluid (CSF) samples from CJD patients essentially as described before [21]. Briefly, ECL-ELISA was performed by overnight coating of carbon ink electrode-embedded microtitre plate with capture antibody at $4{ }^{\circ} \mathrm{C}$. All the coated wells were blocked with $1 \%$ BSA for $1 \mathrm{~h}$ followed by loading of CSF samples (already diluted with $1 \% \mathrm{BSA}=1: 4$ ) in duplicates for $1 \mathrm{~h}$. SULFO-TAG-labelled anti-DJ-1 antibody $(0.1 \mu \mathrm{g} / \mathrm{ml})$ was used as a detection antibody for $1 \mathrm{~h}$ followed by application of $2 \times$ read buffer. Light signal was detected with the help of Meso Scale Discovery Sector 6000 Imager at $620 \mathrm{~nm}$.

\section{Statistical Analysis}

The data were analysed with GraphPad Prism 5 (San Diego, USA). Experimental errors are shown as SEM. Statistical significance was calculated by non-parametric unpaired Student's $t$ test and non-parametric one-way ANOVA followed by Turkey's multiple comparison test. Results were considered significant when $* p<0.05, * * p<0.01$ and $* * * p<0.001$.

\section{Ethics Statement}

Human samples used in this study were obtained from Brain Bank and Biobank of Hospital Clinic-IDIBAPS of the Institute of Neuropathology, HUB-ICO-IDIBELL, Spain, after accomplishing all the rules and laws of Spanish legislation
(Ley de la Investigación Biomédica 2013 and Real DecretoBiobancos, 2014) and also with agreement of the local ethics committees. Mice experiments were conducted in agreement with the ethical regulations of Regierungspräsidium Tübingen (Regional Council) Experimental No. FLI 231/07 file reference number 35/9185.81-2 and also by following the rules and laws of institutional and French national guidelines, in conjuction with the European Community Council Directive $86 / 609 /$ EEC. The experimental protocol was approved by the ethics committee of INRA Toulouse/ENVT.

\section{Results}

\section{Identification of Differentially Expressed Protein Spots by Delta2D Software}

A total of 688 protein spots on all 2DE gels from four independent experiments of MM1 and VV2 subtypes and agematched NDCs were detected and analysed for their differential expression by using $100 \%$ spot matching approach in Delta2D DECODON software. Densitometric analysis revealed 25 significantly differentially altered protein spots (Fig. 1a) which were selected for further identification by using mass spectrometry.

\section{Identification of Differentially Expressed Proteins}

Identification with Q Exactive hybrid quadrupole/orbitrap mass spectrometry revealed 366 proteins in both subtypes. Screening by matching the $p I$ value and molecular weight of each identified protein to its corresponding spot unveiled 83 expressionally altered proteins in both subtypes. Forty proteins in MM1 and 43 proteins in VV2 subtype were identified

Fig. 1 Identification of differentially regulated protein spots by Delta2D software and differentially regulated proteins by mass spectrometry in cerebellum of MM1 and VV2 subtypes of SCJD. a Map of silverstained $2 \mathrm{DE}$ gel showing 25 differentially regulated protein spots identified with the help of Delta2D software after warping of all protein spots on all gels from both MM1 and VV2 subtypes of sCJD from cerebellum of brain is shown along with their $p I$ values and molecular weights. These spots had more than 1.5 -fold change and $p$ value $<0.05$ calculated by unpaired Student's $t$ test. b Number of proteins identified by Q Exactive hybrid quadrupole/orbitrap mass spectrometry in MM1 and VV2 subtypes of SCJD. Mass spectrometry of 25 differentially regulated protein spots revealed 366 regulated proteins in cerebellum of MM1 and VV2 subtypes of SCJD. Only 83 proteins of total identified proteins with minimal peptide count of 2 qualified further screening criteria of $p I$ and molecular weight match to that of their corresponding spots. Forty and 43 proteins were regulated in MM1 and VV2 subtype each respectively with 12 proteins common between two subtypes. c Expression of 18 proteins was increased, and 22 were decreased in MM1 subtype, whereas expression of 25 proteins was increased, and 18 were decreased in VV2 subtype of SCJD cases. Upregulated proteins are shown by red and downregulated proteins by yellow bars 
a

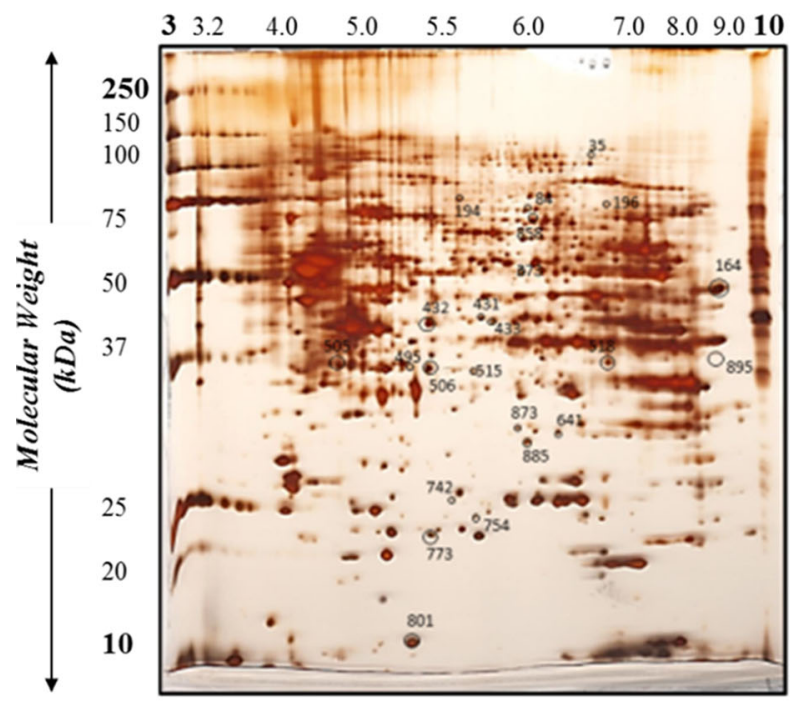

C

\section{Regulated Proteins in MM1 subtype of SCJD}

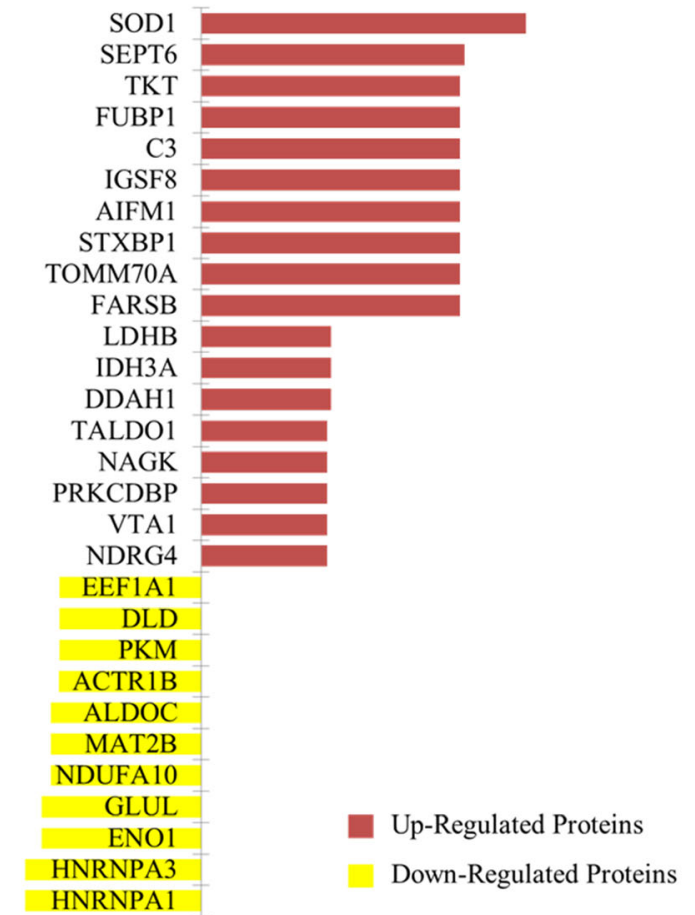

b

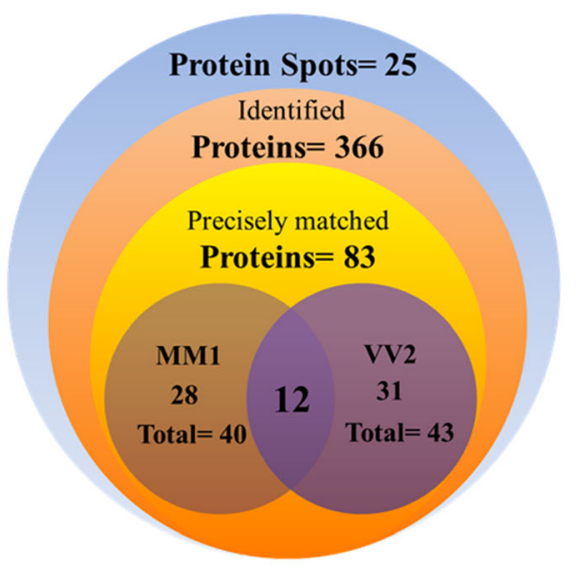

Regulated Proteins in VV2 subtype of SCJD

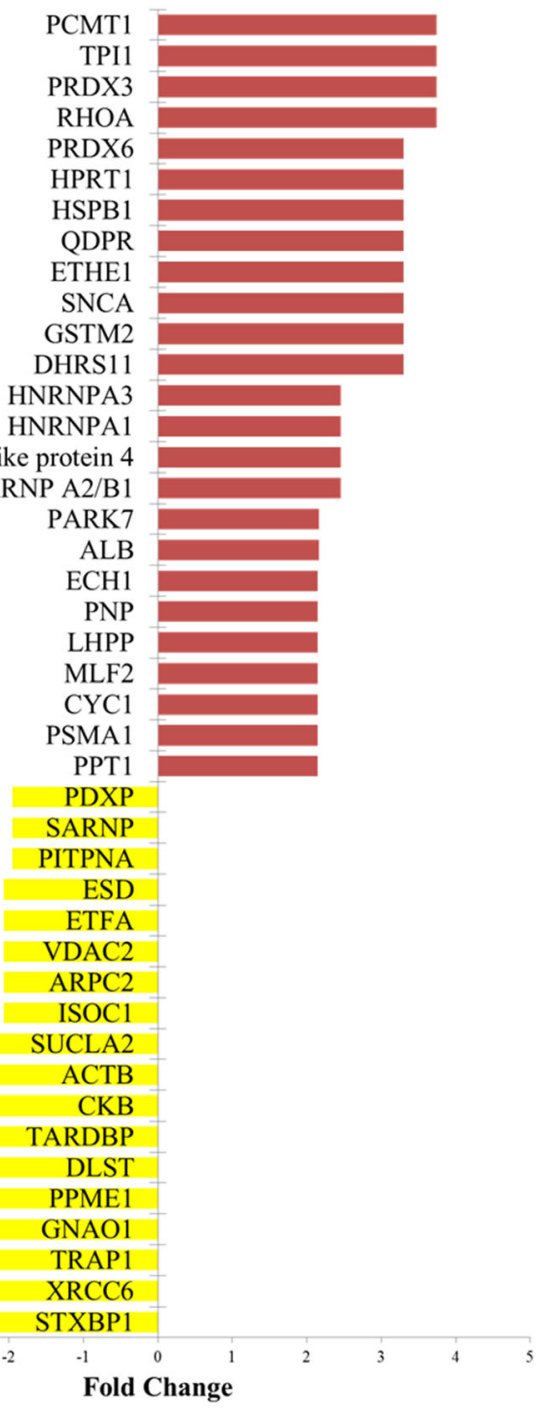


to be differentially expressed with 12 of those common between the two subtypes (Fig. 1b). In MM1 subtype, 18 proteins showed an increased expression and 22 showed a decreased expression, whereas in VV2 subtype, 25 proteins showed an increased expression and 18 showed a decreased expression as compared to age-matched NDCs (Fig. 1c).

Further, sCJD-related proteins in both subtypes showed almost similar trend of expressional alteration (either increased or decreased) between MM1 and VV2 subtypes. Twelve proteins were identified commonly in both (MM1 and VV2) subtypes with seven of them showing decrease in their expression in both subtypes and were including guanine nucleotide-binding protein $\mathrm{G}(\mathrm{o})$ subunit alpha, protein phosphatase methylesterase 1, dihydrolipoyllysine residue succinyltransferase component of 2-oxoglutarate dehydrogenase complex, TAR DNA-binding protein 43, creatine kinase B-type, actin, cytoplasmic 1 and succinylCoA ligase [ADP-forming] subunit beta. Five proteins showed subtype-specific expressional alteration. Expression of syntaxinbinding protein 1 was increased in MM1 subtype but decreased in VV2 as compared to their age-matched NDCs. Expression of dihydrolipoyl dehydrogenase, heterogeneous nuclear ribonucleoproteins A2/B1, ELAV-like protein 4, heterogeneous nuclear ribonucleoprotein $\mathrm{A} 3$ and heterogeneous nuclear ribonucleoprotein A1 was increased in VV2 subtype in comparison to MM1 and vice versa in MM1 subtype in comparison to VV2.

All differentially expressed identified proteins from both SCJD subtypes were grouped on the basis of their molecular functions into eight groups (Table 1) which include (i) cell cycle, gene expression and cell death; (ii) cellular stress response/oxidative stress; (iii) signal transduction and synaptic functions, (iv) carbohydrate metabolism; (v) energy metabolism; (vi) cell growth, proliferation and differentiation and (vii) intracellular transport and cytoskeleton-related functions. A few proteins which could not be classified into any of the abovementioned groups were classified under other functions.

\section{Regulation of Identified Cellular Molecular Mechanisms}

The functional classification of all identified proteins in SCJD (MM1 and VV2) subtypes revealed overall alteration of nearly all cellular pathways during the course of the disease (Fig. 2a, b). Cell cycle, gene expression and cell death-related cellular molecular functions were 24\% in MM1 and 27\% in VV2 subtype, cellular stress response/oxidative stress-related cellular functions $22 \%$ in MM1 and 25\% in VV2 subtype and signal transduction and synaptic functions 15\% in MM1 and 18\% in VV2 subtype altered based on the number of expressionally altered proteins to the corresponding molecular function and were also the three most altered cellular functions in both subtypes.

Most of the proteins involved in cellular stress response/ oxidative stress-related functions in both SCJD subtypes were related to oxidative stress. Similarly, other molecular functions including carbohydrate metabolism were $11 \%$ in
MM1 and 2\% in VV2 subtype, energy metabolism 9\% in MM1 and 7\% in VV2 subtype, cell growth, proliferation and differentiation-related cellular molecular functions $6 \%$ in MM1 and 5\% in VV2 subtype and intracellular transport and cytoskeleton-related molecular functions $9 \%$ in MM1 and $7 \%$ in VV2 subtype altered. Of proteins from MM1 and VV2 subtypes, 4 and $9 \%$ respectively were not able to be assigned any function, so they were subclassified under 'other' functions.

Functions of identified regulated proteins in both subtypes are mentioned as supporting information in file 3.

\section{Validation of MS/MS Data}

Eleven proteins from whole proteomic data set identified by MS/MS were selected, based on low and high peptide counts, sequence coverage and fold change, both downregulated as well as upregulated ones, to validate their expressional alteration at mRNA level (Fig. 3a) and at protein level (Fig. 3b, c) in order to minimize any perplexity in the proteomic data. Expressional alteration of corresponding protein spots selected for validation of proteomic findings after mass spectrometry is shown in supplementary Fig. 3.

\section{Validation at mRNA Level by RT-qPCR}

During the analysis of mRNA expression profiling (Fig. 3a), pyruvate kinase M2 showed no alteration in its mRNA expression in MM1 but a significant increase in VV2 subtype. The mRNA expression for septin-6 was significantly increased in both subtypes (MM1 and VV2) and for TAR DNA-binding protein $43(T D B P)$ only in MM1 but unaltered in VV2 subtype. A significant decrease in mRNA expression of creatine kinase B type $(C K B)$ was observed only in MM1 but no alteration in VV2 subtype. Succinyl-CoA ligase [ADP-forming] subunit beta (SUCLA-2) and L-lactate dehydrogenase B $(L D H B)$ showed unaltered mRNA expression in both subtypes (MM1 and VV2). Transaldolase (TALDO-1) showed a significant increase in its mRNA expression in both subtypes (MM1 and VV2), and peroxiredoxin-6 (PRDX$6)$ showed a significant increase in its mRNA expression only in VV2 subtype. Whereas, heat shock protein beta-1 (HSPB-1) and protein DJ-1 (PARK-7) showed a significant increase at mRNA level in both subtypes (MM1 and VV2). The mRNA expression of superoxide dismutase $[\mathrm{Cu}-\mathrm{Zn}](S O D-1)$ was also found significantly increased in both subtypes (MM1 and VV2) (Supplementary Fig. 2C). Altogether, these results showed that mRNA expression of mostly validated proteins showed similar trend to their corresponding regulated protein spots except unchanged for $L D H B, S U C L A-2$ 


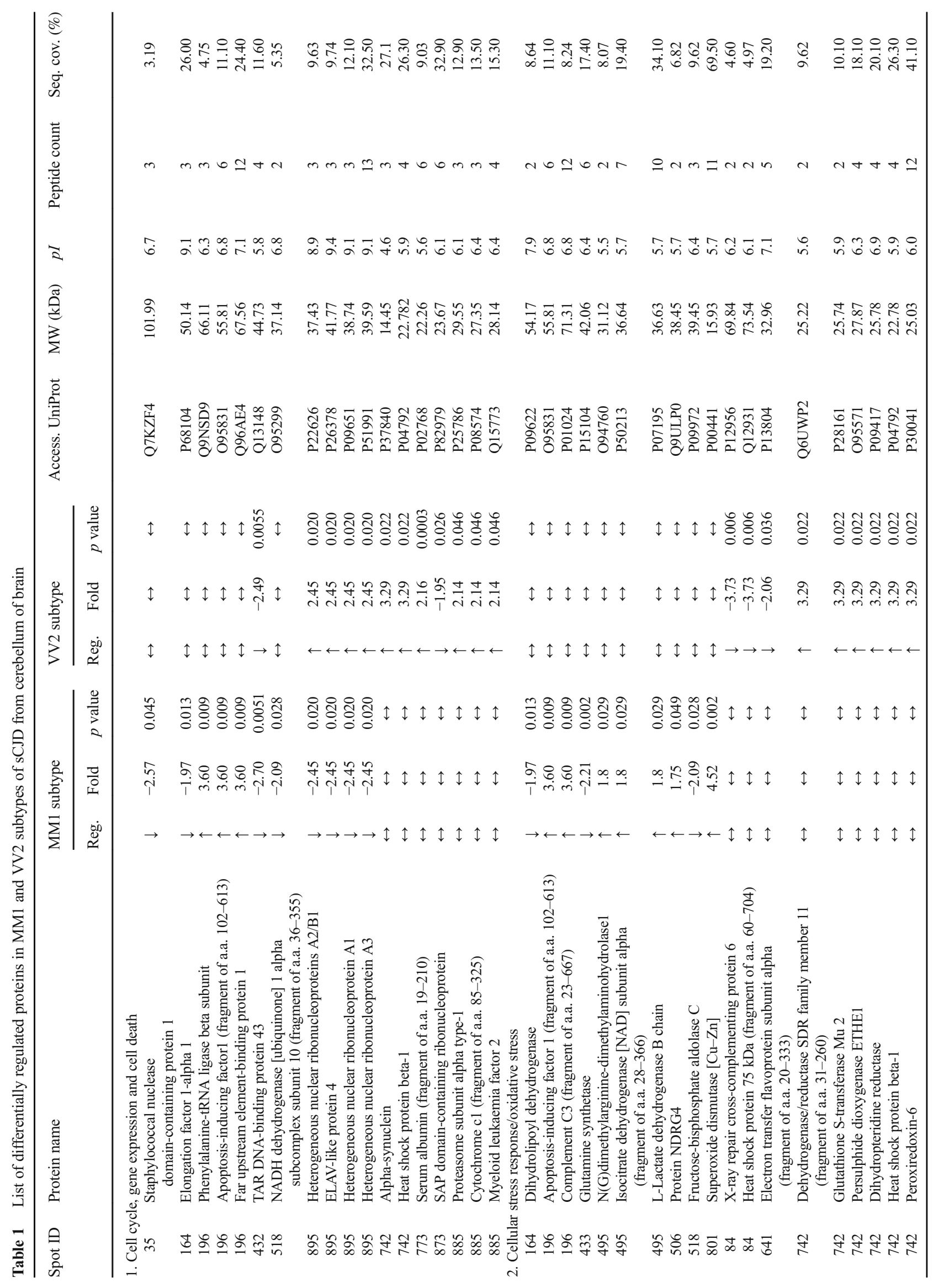




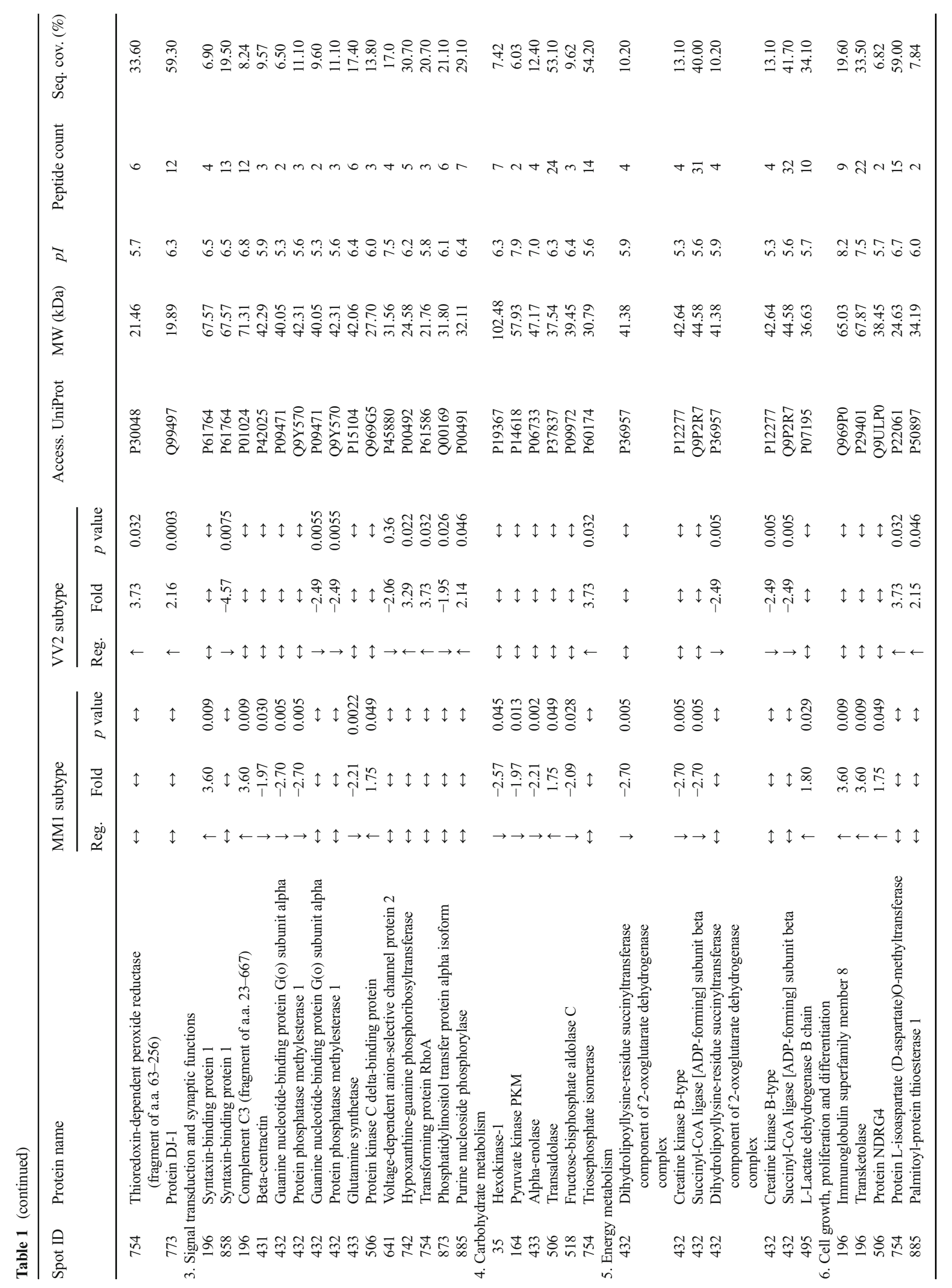




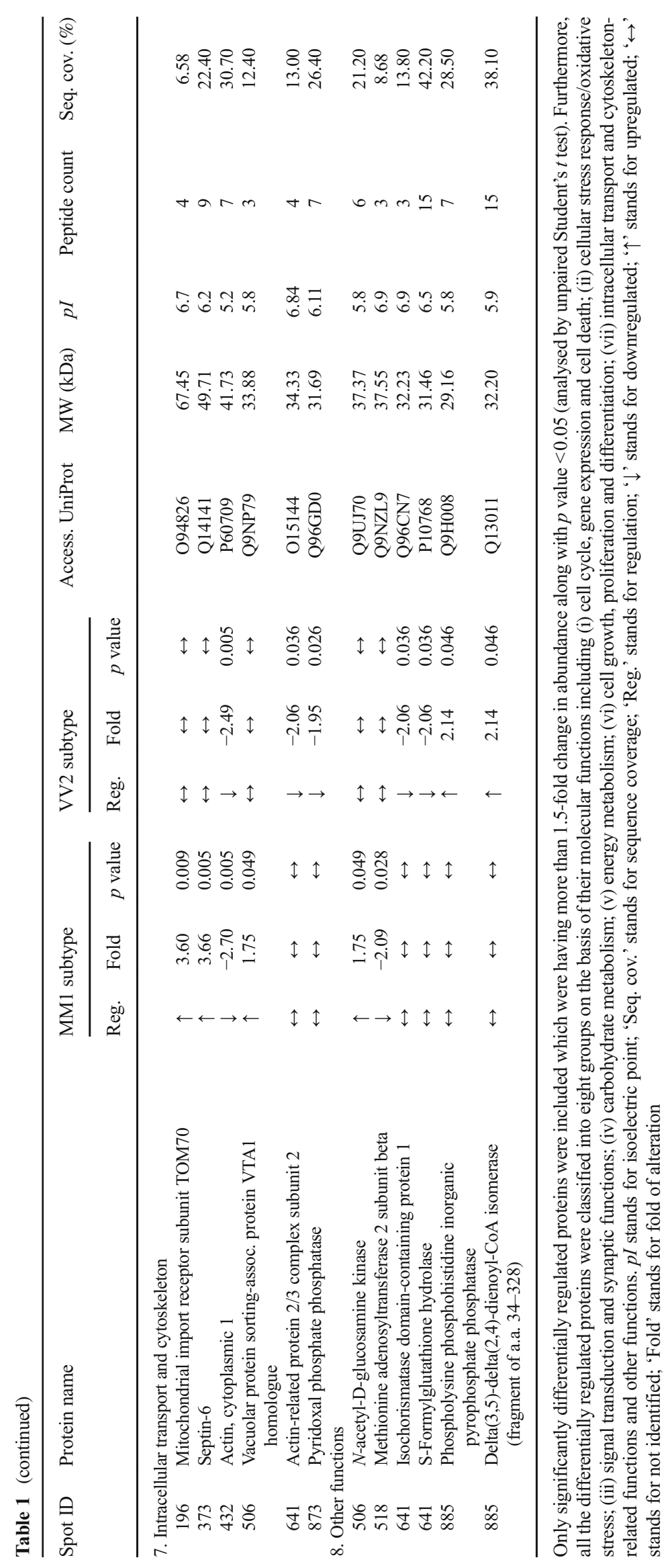




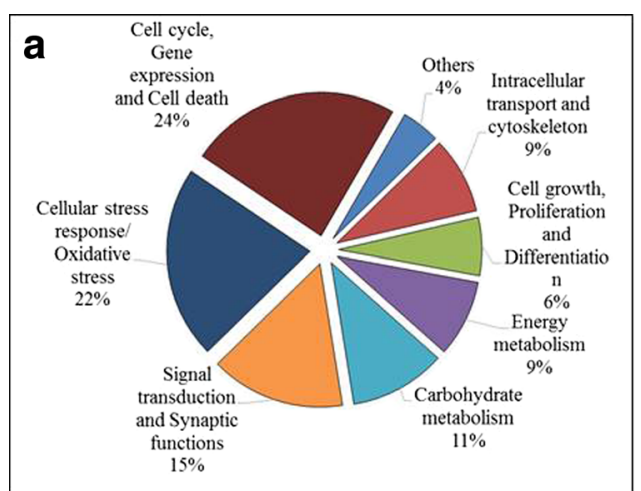

Fig. 2 Identification of regulated molecular functions in MM1 and VV2 subtypes based on classification of identified proteins into different functional groups: All identified proteins were further classified into different functional groups based on their molecular functions showing

and $T D P(\mathrm{VV} 2)$ while increased for $P K M-2$ (in VV2) and $T D P$ (in MM1).

\section{Validation at Protein Level by Western Blot}

Already validated proteins at their mRNA level were further validated for their expressional alteration at protein level by using western blot (Fig. 3b, c). Densitometry analysis revealed a significant decrease of protein expression for PKM2, TDP43 and SUCL-2 only in MM1 subtype and for CKB in both (MM1 and VV2) subtypes. Although PKM2 and TDBP showed the trend of a decrease in their protein expression in VV2 subtype, yet results were not statistically significant. A significant increase of protein expression was found for $\mathrm{LDH}-$ B, HSP beta 1 and Prdx6 in both subtypes (MM1 and VV2) and for SEPT6 and DJ-1 only in VV2 subtype. TALDO1 did not show any detectable alteration in western blotting. SOD1 also showed a significant increase in its protein expression in both subtypes (MM1 and VV2) (Supplementary Fig. 2C).

In summary, these results show that protein expression of all the validated proteins except TALDO1 showed a similar trend of alteration to their corresponding regulated protein spots identified later by MS/MS.

\section{Identification of DJ-1 During the Pathophysiology of SCJD}

Proteins having highest fold changes, peptide counts and sequence coverages in our data set belonged mainly to either metabolic pathways or oxidative stress-related functions. Amongst many oxidative stress-related identified proteins, DJ-1 had the highest peptide count and the second heighest sequence coverage indicating a very low false discovery rate (FDR) and very high confidence of its true identifaction in SCJD. DJ-1 is known for its protective role against oxidative stress and as a sensor of oxidative stress. Therefore, further

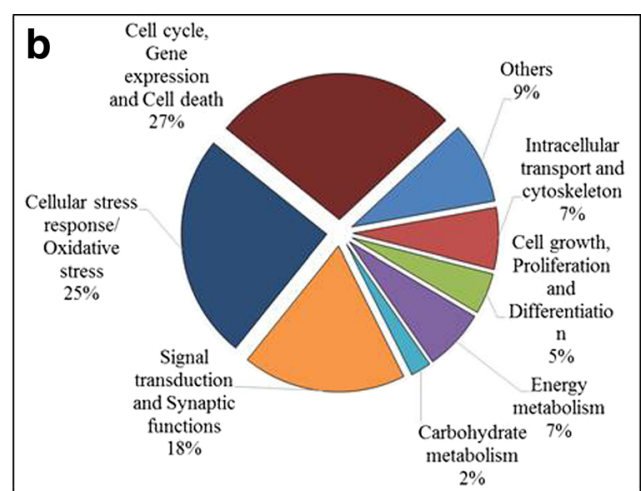

the \% regulation of different molecular functions in a MM1 and b VV2 subtype. The molecular functions were assigned as in UniProt protein database

experiments were performed to elucidate the implication of DJ-1 during the pathophysiology of the disease in SCJD.

\section{Expressional Alteration of DJ-1 in Cerebellum of $s C J D$}

Immunofluorescent experiments were performed to highlight if expressional alteration of DJ-1 is due to oxidative stress specifically in cerebellum of SCJD. DJ-1 is known to translocalize to the mitochondrial matrix [22] and nucleus [23] during oxidative stress. Results of immunofluorescent experiment showed a significant increase in the expression of DJ-1 in the Purkinje cell layer as well as in granular cell layer in cerebellum of SCJD (MM1) sections as compared to age-matched NDCs. Further, DJ-1 expression was increased in the nuclear region of granular cell layer of cerebellum of sCJD (MM1) sections as compared to age-matched NDCs showing enhanced nuclear translocalization (Fig. 4a). These results suggested that expressional alteration of DJ-1 in SCJD could be linked to oxidative stress.

Fig. 3 Validation of selected proteins at transcriptional and translational levels: a RT-PCR analysis to validate mRNA expression of PKM2, SEPT-6, TDBP, CKB, SUCLA-2, LDHB, TALDO1, HSPB1, Prdx6 and PARK7 in control $(n=14 \pm 1), \operatorname{sCJD}$ MM1 $(n=14 \pm 1)$ and SCJD VV2 $(n=9 \pm 1)$ samples is shown. Values are normalized using GUS-b as internal controls. The statistical significance was calculated with one-way ANOVA followed by Turkey post test to compare all pairs of columns. Data are represented as the mean SEM. $* p<0.05$, $* * p<0.005$, $* * * p<0.001$. b Western blot analysis to validate protein expression of PKM2, SEPT6, TDP43, CKB, SUCL-2, LDH-B, TALDO1, HSPB1, Prdx6 and DJ-1 in control $(n=4)$, sCJD MM1 $(n=4)$ and sCJD VV2 $(n=4)$ samples is shown. GAPDH is used as a loading control. c Densitometeric analysis of PKM2, SEPT6, TDP43, CKB, SUCL-2, LDH-B, TALDO1, HSPB1, Prdx6 and DJ-1 with control $(n=4)$, sCJD MM1 $(n=4)$ and sCJD VV2 $(n=4)$ samples is shown. The statistical significance was calculated with one-way ANOVA followed by Turkey post test to compare all pairs of columns. Data are represented as the mean SEM. $* p<0.05, * * p<0.005, * * * p<0.001$ 
a

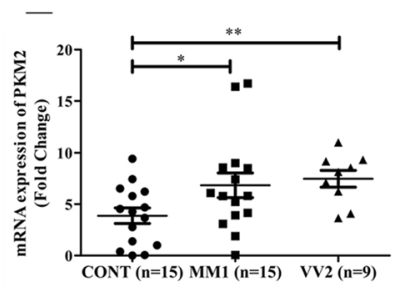

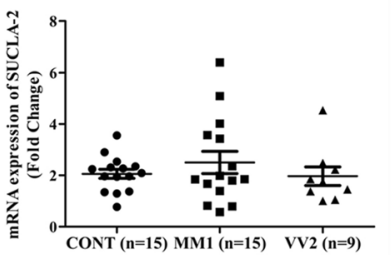

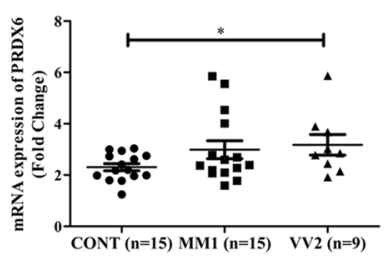

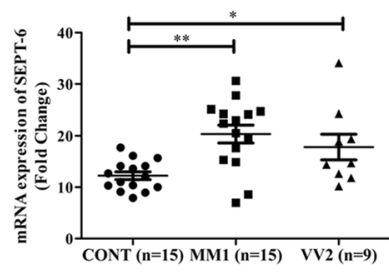
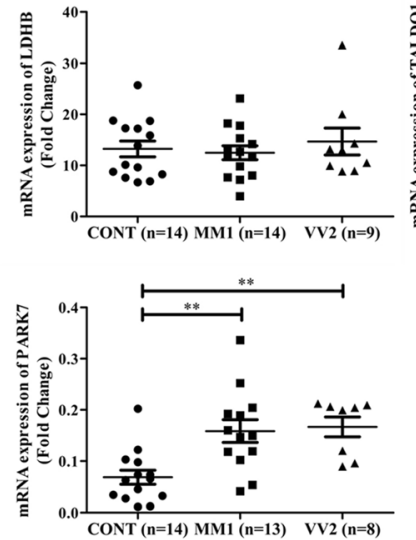

b

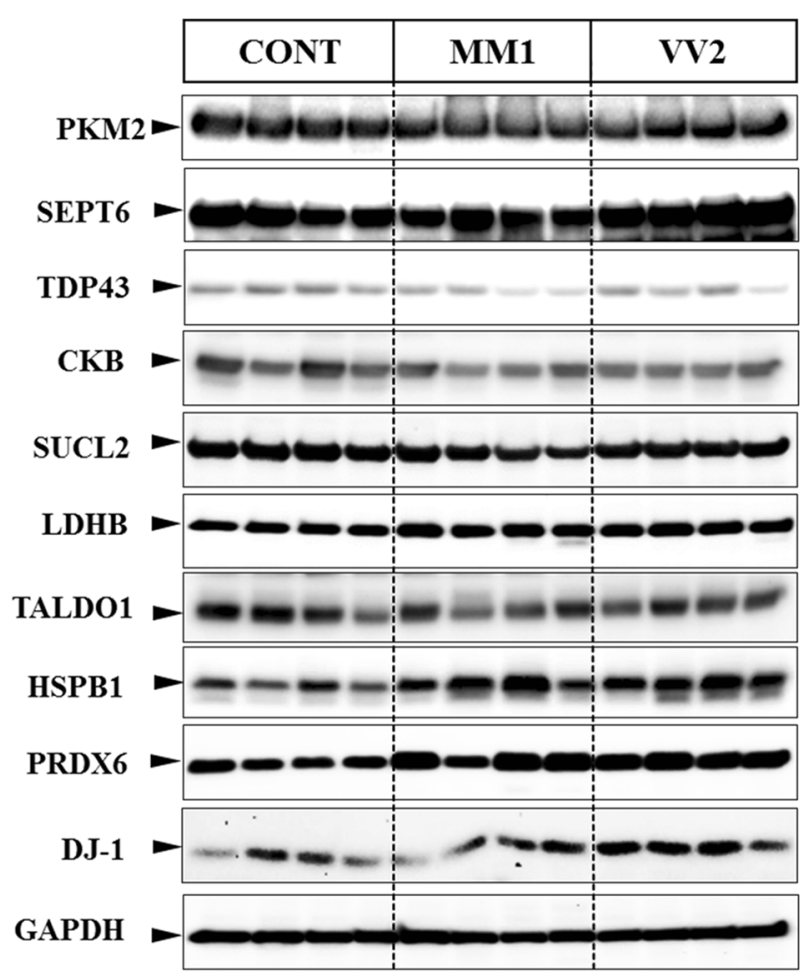

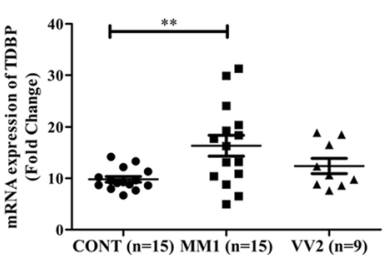
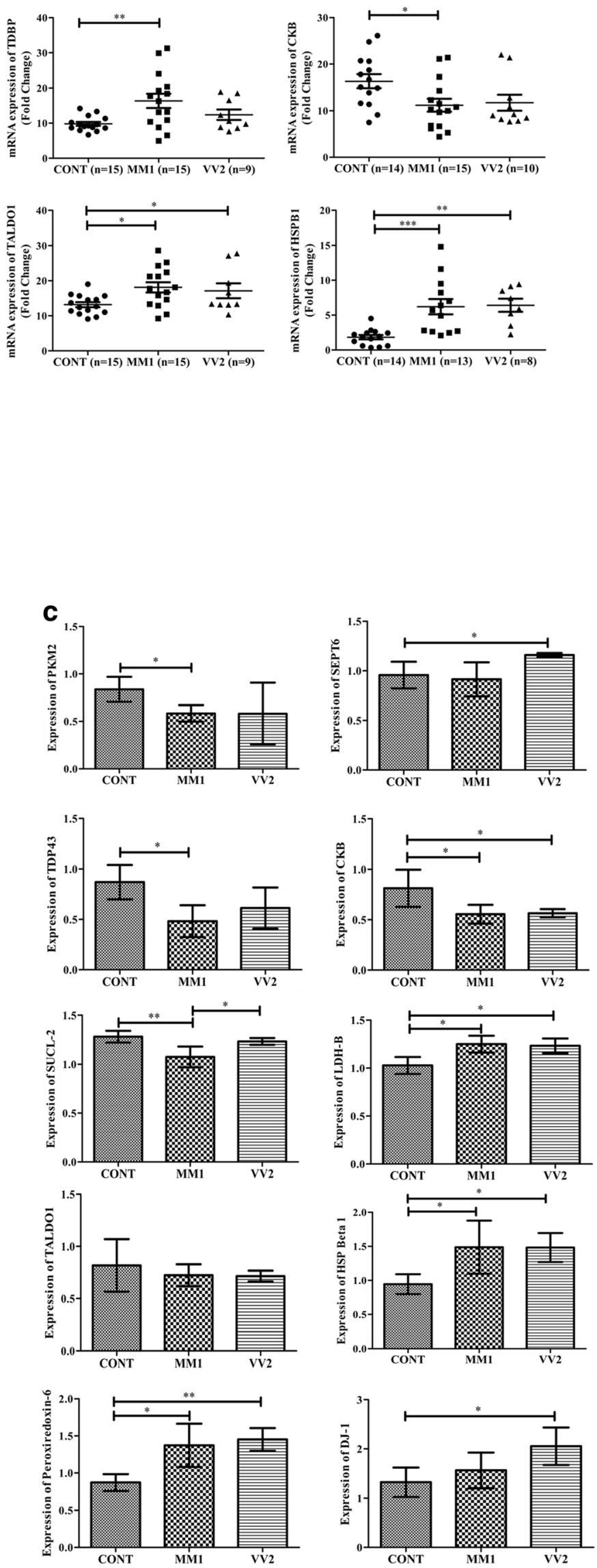
a

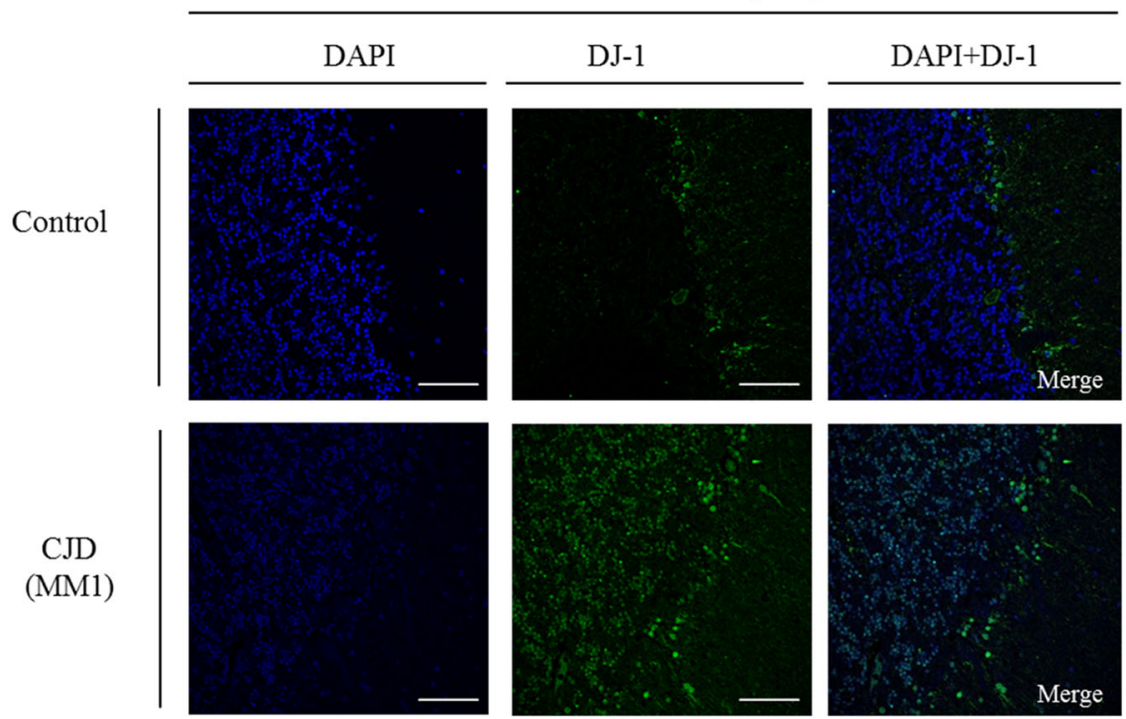

b

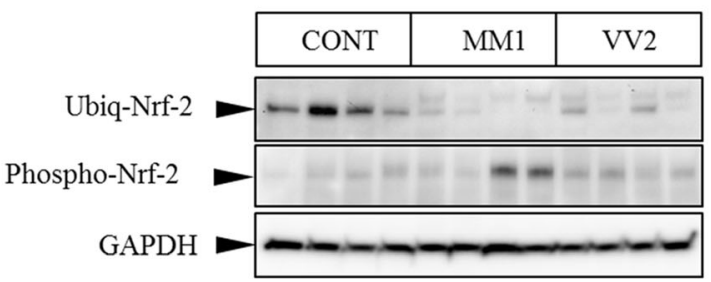

mRNA expression of Nrf2 Protein expression of Nrf2 Protein expression of P-Nrf2

P-Nrf2/Nrf2 Ratio
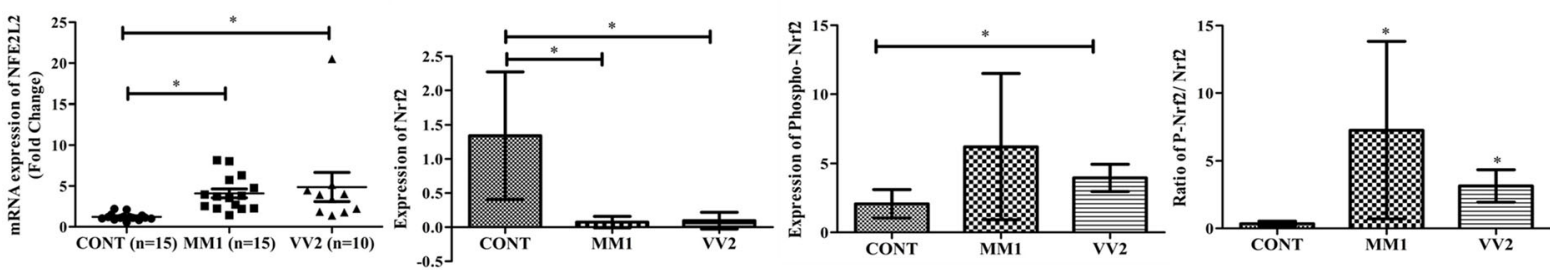

C

$$
\text { HMOX1 }
$$

PRDX3
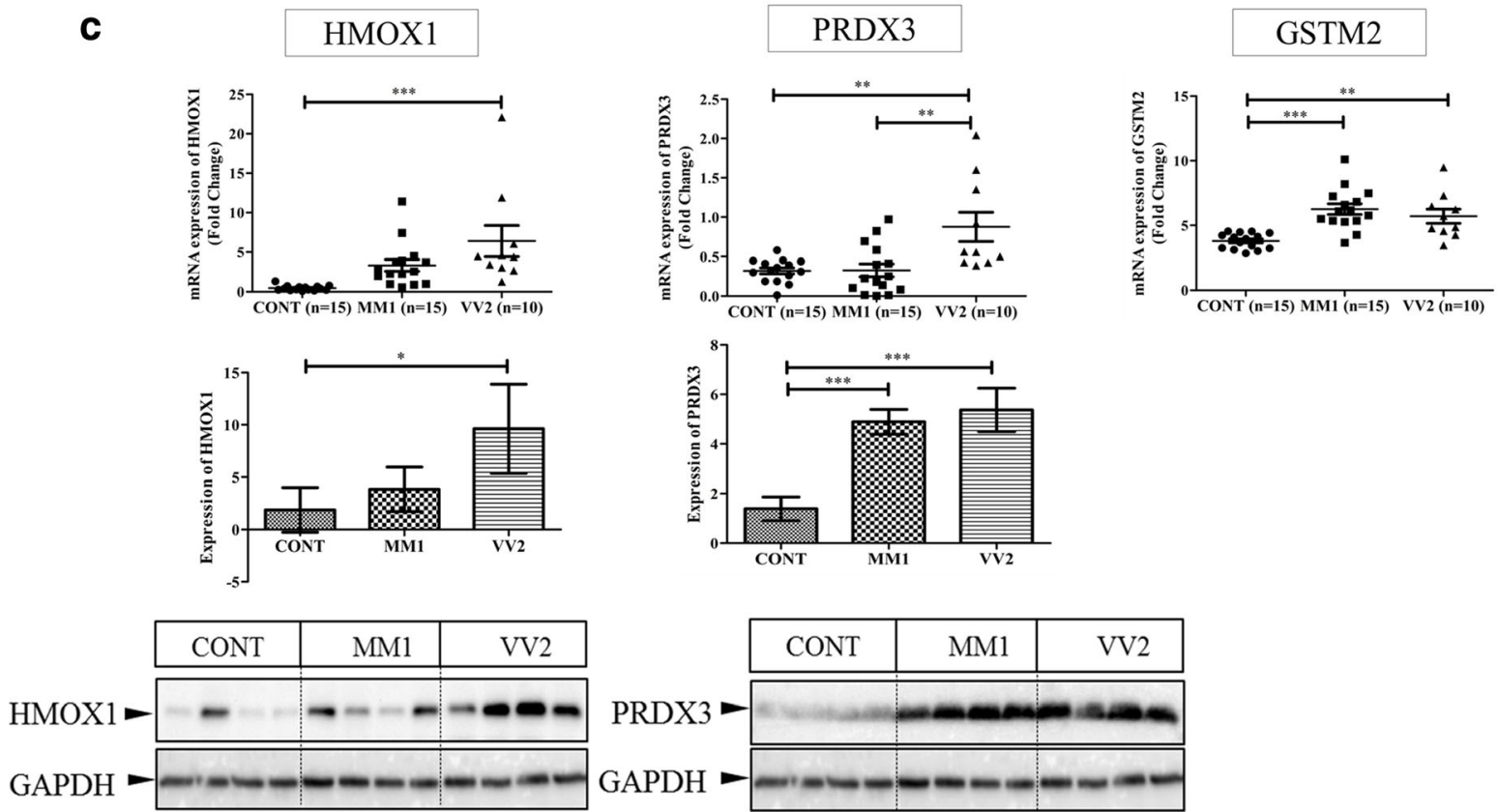
4 Fig. 4 Expressional alteration of DJ-1 in cerebellum of sCJD (MM1) and further implication of DJ-1 in mediating Nrf2/ARE pathway as its downstream regulatory effects: DJ-1 is reported to translocalize to nucleus under the effect of oxidative stress. a Immunoflourescence experiment results showed an increase in DJ-1 expression and its oxidative stress-mediated nuclear translocalization in granular cell layer of cerebellum of human SCJD MM1 as compared to age-matched NDCs using anti DJ-1 primary antibody which confirms that expressional alteration of DJ-1 is due to oxidative stress. $\mathbf{b} \mathrm{Nrf} 2$ is a transcriptional regulator which is reported to be regulated by DJ-1 during oxidative stress. After its activation by DJ-1, Nrf2 is phosphorylated which is then translocalized to nucleus to activate the antioxidative response elements (AREs). Expressional analysis of $\mathrm{Nrf} 2$ revealed an increased mRNA expression of NFE2L2 in cerebellum of both MM1 $(n=15)$ and VV2 $(n=10)$ as compared to age-matched NDC ( $n=15)$ using qPCR. Values are normalized using GUS-b as internal control. Western blotting of samples from MM1 $(n=4)$ and VV2 $(n=4)$ subtypes of SCJD along with age-matched NDC $(n=4)$ from cerebellum with anti-Nrf2 and anti-phospho-Nrf2 antibody is also shown. GAPDH was used as a loading control. Densitometeric analysis of western blot results showed a significant decrease in ubiquinated Nrf2 expression in both MM1 and VV2 subtypes and increased expression of phospho-Nrf2 in VV2 subtype of sCJD. Additionally, P-Nrf2/Nrf2 ratio was also calculated and found significantly increased in both MM1 and VV2 subtypes of SCJD as compared to age-matched NDCs. The statistical significance was calculated with one-way ANOVA followed by Turkey post test to compare all pairs of columns. Data are represented as the mean SEM. ${ }^{*} p<0.05, * * p<0.005, * * * p<0.001$. Phospho-Nrf2 (the activated form of Nrf2) activates hemeoxygenase 1 (HO-1), thioredoxin-depedent peroxide reductase (PRDX3) and glutathione S-transferase Mu 2 (GSTM2) in the nucleus which are the members of AREs. c mRNA expressions of HMOX-1, PRDX3 and GSTM2 in MM1 $(n=15)$, VV2 $(n=10)$ and age-matched NDC $(n=10)$ was detected by using qPCR. Values are normalized using GUS-b as internal control. Further protein expression of HO-1 and PRDX3 estimated with western blotting of samples from MM1 $(n=4)$ and VV2 $(n=4)$ subtypes of sCJD and age-matched NDC $(n=4)$ from cerebellum using anti HO-1 and anti PRDX3 antibodies is also shown along with their densitometeric analysis. GAPDH was used as a loading control. GSTM2 was identified by MS/MS in protein spot 742 whose spot intensity was found significantly increased in VV2 subtype of sCJD samples indicating an increase in GSTM2 protein expression in VV2 subtype of sCJD samples. The statistical significance was calculated with one-way ANOVA followed by Turkey post test to compare all pairs of columns. Data are represented as the mean SEM. $* p<0.05, * * p<0.005$, $* * * p<0.001$

\section{Regulation of Nrf2/Antioxidative Response Element Pathway in Cerebellum of $S$ CJD (MM1 and VV2) Subtypes}

One proposed cellular mechanism of action of DJ-1 in regulating the oxidative stress is by regulation of $\mathrm{Nrf} 2$ signalling pathway [24]. Nrf2 is a transcription factor and involved in regulating the expression of antioxidative proteins for protection against oxidative stress implied by ROS or inflammation [25]. Nrf2 showed a significant increase in its mRNA expression both in MM1 and VV2 subtypes of sCJD cases indicating high cellular demand for Nrf2. Under normal physiological conditions, Nrf2 is continuously ubiquitinated in the cytoplasm for degradation by proteasomes as its regulatory mechanism. We observed a significant decrease in the expression of ubiquitinated Nrf2 in MM1 and VV2 subtypes of sCJD as compared to age-matched NDCs (Fig. 4b). After release of Nrf2 from Keap1, it is phosphorylated. An increase in the expression of phosphorylated Nrf2 was observed in both MM1 and VV2 subtypes of SCJD as compared to agematched NDCs, but its expression was significantly increased only in VV2 subtype (Fig. 4b. Additionally, P-Nrf2/Nrf2 ratio was also found significantly increased in both MM1 and VV2 subtypes of sCJD as comapared to age-matched NDCs (Fig. 4b).

After translocation into the nucleus, phosphorylated Nrf2 is reported to induce the transcription of ARE (NADPH quinone peroxidase, heme oxygenase 1 (HMOX-1), glutathione Stransferase (GST), peroxiredoxins (PRDX), glutathione cysteine ligases (GCLs) and glutathione peroxidase) in the nucleus $[25,26]$. We examined the expressional profile of some selective members of ARE family including HMOX-1, PRDX3 and GSTM2 in MM1 and VV2 cases of sCJD (Fig. 4c). HO-1 and PRDX3 showed a significant increase in mRNA expression only in VV2 subtype, while GSTM2 showed a significant increase in mRNA expression in both MM1 and VV2 subtypes as compared to their healthy NDCs, whereas at translational level, HO-1 showed a significant increase in VV2 subtype and PRDX3 showed a significant increase in both MM1 and VV2 subtypes of sCJD. GSTM2 was identified by MS/ MS in protein spot 742 which was found significantly increased in VV2 subtype of sCJD samples indicating an increase in GSTM2 protein expression also in VV2 subtype of sCJD samples. These results indicate the activation of ARE machinery in cerebellum of sCJD (MM1 and VV2).

\section{Expressional Alteration of DJ-1 During the Clinical Phase in SCJD Patients}

CSF reflects the pathophysiological events taking place inside the brain. To explore the expressional alteration of DJ-1 during the clinical phase in human SCJD cases, protein concentration of DJ-1 was examined in clinical CSF samples of SCJD (MM1 and VV2) patients with ECL-based ELISA. Protein concentration of DJ-1 was found to be significantly increased in both SCJD (MM1 and VV2) subtype cases as compared to age-matched NDC (Fig. 5) indicating the secretion of DJ-1 from brain tissue into CSF during the clinical phase of SCJD (MM1 and VV2) subtypes.

\section{Expressional Alteration of DJ-1 During the Progression of the Disease in CJD}

In order to evaluate the expressional alteration of DJ-1 during the progression of disease in CJD, transgenic mice inoculated with SCJD (MM1 and VV2) brain tissue were used. 


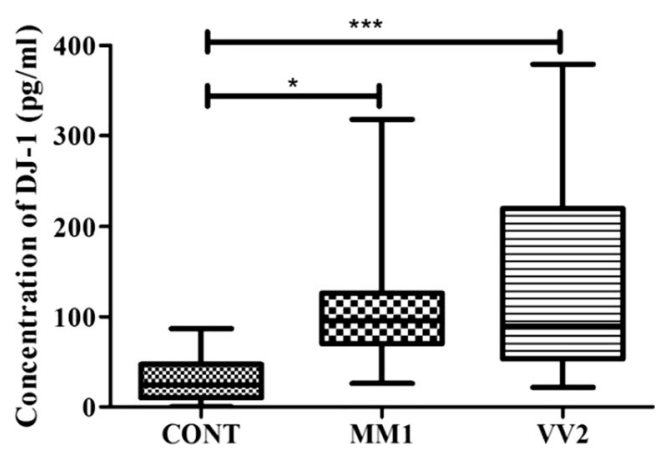

Fig. 5 Expressional alteration of DJ-1 during the clinical phase of the disease in CSF samples of sCJD (MM1 and VV2) patients: Analysis of DJ-1 expression with the help of highly sensitive ECL-based ELISA in clinical CSF samples of healthy control $(n=18), \operatorname{SCJD}$ MM1 $(n=18)$ and sCJD VV2 $(n=18)$ is shown. The statistical significance was calculated with one-way ANOVA followed by Turkey post test to compare all pairs of columns. $* p<0.05, * * p<0.005, * * * p<0.001$

Immunofluorescent experiments with cerebellum of SCJD (MM1)-inoculated transgenic mice along with their agematched NDC showed a significant increase in the expression of DJ-1 in the cells of Purkinje cell layer as well as in granular cell layer in cerebellum sections of CJD (MM1) mice as compared to controls. Furthermore, DJ-1 expression was increased in the nuclear region of granular cell layer of cerebellum sections of CJD (MM1) mice showing enhanced nuclear translocalization as compared to healthy controls (Fig. 6a). These results suggested that expressional alteration of DJ-1 in cerebellum of SCJD (MM1)-inoculated transgenic mice could be linked to oxidative stress like in human SCJD cases as described previously in "Expressional Alteration of DJ-1 in Cerebellum of sCJD" section.

\section{Expressional Alteration of DJ-1 at Presymptomatic and Symptomatic Stages in CJD Mice}

Western blot analysis showed a significant increase in the expression of DJ-1 in the cerebellum of sCJD MM1 mice at presymptomatic stage as compared to age-matched healthy controls. DJ-1 expression was decreased during early symptomatic stage but again started to rise significantly during late symptomatic stage in MM1 subtype as compared to its expression at presymptomatic stage (Fig. 6b). But expression of DJ1 remained higher during the course of the disease right from the beginning of disease progression as compared to the corresponding healthy controls. Similarly, DJ-1 expression in the cerebellum of sCJD VV2 mice was also significantly increased during early symptomatic stage as compared to healthy controls, whereas DJ-1 expression was decreased during both symptomatic and late symptomatic stages as compared to its expression at presymptomatic stage (Fig. 6b). But expression of DJ-1 remained higher throughout the progression of disease as compared to corresponding healthy controls.

\section{Discussion}

In order to explore the contributing pathophysiological factors, identification of proteome-wide alterations during the course of disease can be helpful in adding more information to the existing knowledge. Results in this study showed proteomic differences between the two subtypes (MM1 and VV2) highlighting that the origin of pathological features between two subtypes is PrP codon 129 genotype dependent. Subtype and regional-dependent differential alterations of spongiosis, neuroinflammation, gliosis, synaptic damage and intracellular vesicular transport between MM1 and VV2 subtypes in the brains of SCJD patients have been already reported which highlight that PrP codon-129 genotype could be involved also in the differential selection of the region of brain for the differential pathological ailments to occur [27-32]. So, differential $\operatorname{PrP}^{\mathrm{Sc}}$ aggregates, deposition, neuronal as well as synaptic loss in the cerebellum of VV2 subtype $[2,33]$ could be responsible for severe pathological outcomes and molecular alterations in VV2 subtype than in MM1 subtype in this study. On the contrary, when compared with the frontal cortex region of SCJD patients, molecular functions like signal transduction and neuronal activity, cell structure and motility and protein metabolism appeared to be more altered in MM1 subtype as compared to VV2 subtype [4], supporting the differential pathological features in different regions of the brain determined by PrP codon 129 genotype.

Proteomic findings in this study indicated that nearly all the cellular molecular functions are impaired during the pathophysiology of the disease. A diverse number of mechanisms and proteins regulating gene expression were also identified which could be involved in dysregulating gene expression in the pathophysiology of sCJD. Cell cycle and gene expressionrelated functions were the top most altered molecular functions in both subtypes (24\% in MM1 and 27\% in VV2) in this study. Protein expression could be altered due to regulation of pre-mRNA processing involving its packing into heterogeneous nuclear RNA (hnRNA), half-life and transport to cytoplasm. Dysregulations in RNA processing and, in particular, the aberrant regulation of RBPs have been reported to play a basic role in the pathogenesis of neurodegenerative diseases [27, 34-39]. Proteins including heterogeneous nuclear ribonucleoproteins A2/B1, heterogeneous nuclear ribonucleoprotein A1, heterogeneous nuclear ribonucleoprotein A3, SAP domain-containing ribonucleoprotein, ELAV-like protein 4 and TAR DNA-binding protein 43 identified to be altered in this study could be responsible for proteomic alterations by regulating mRNA processing and expression [40]. hnRNP have been implicated in several neurodegenerative diseases, mitochondrial dynamics and impaired cognition in Alzheimer's disease (AD) [41-43]. ELAV proteins have been implicated in the regulation of HO-1 expression at both mRNA and protein levels [44]. Proteins including 
a Mice Cerebellum (MM)

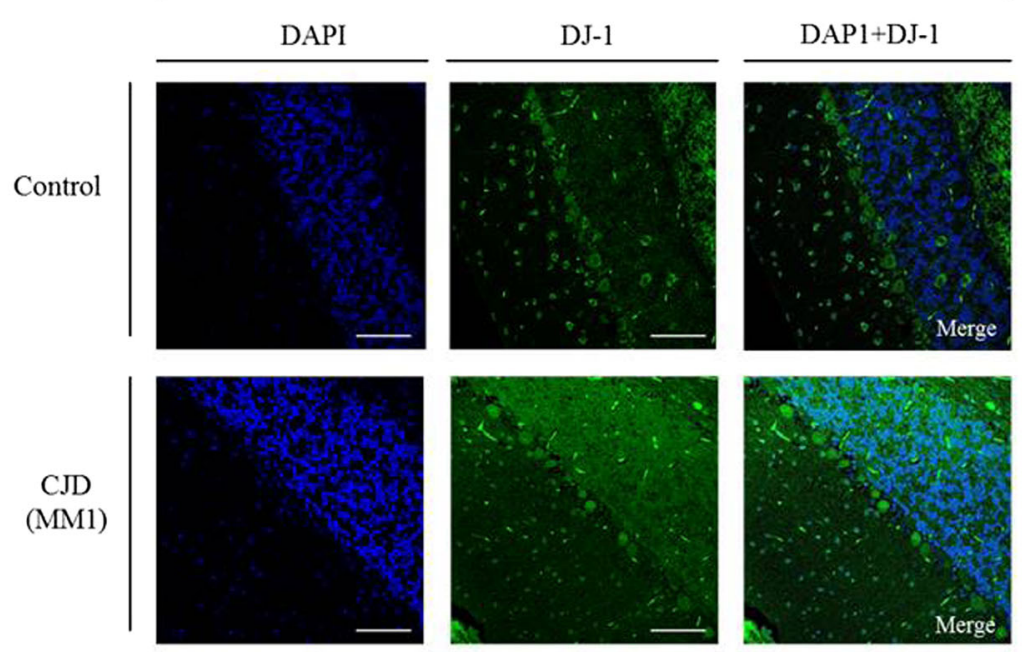

b
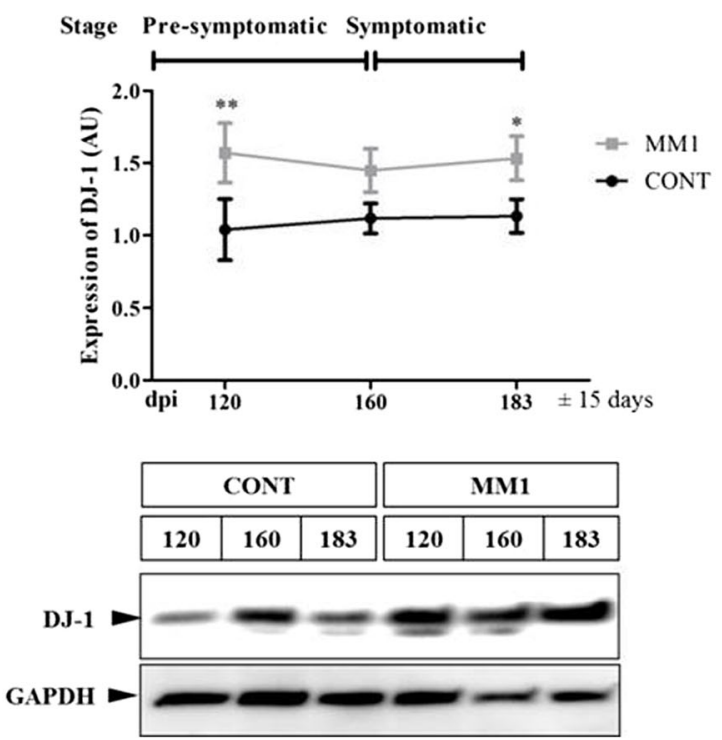

Fig. 6 Expressional alteration of DJ-1 during the progression of the disease in SCJD MM1 and VV2 transgenic mice: Double transgenic mice Tg340 knockout for PrP and expressing 4-fold expression of humanized PrP with either methionine or valine at codon 129 of $\operatorname{PrP}$ were inoculated with $10 \%$ brain homogenate from sCJD patient either with MM1 or VV2 subtype of SCJD. Mice were sacrificed, and brain samples were collected at 120,160 and 183 days post inoculation (dpi) for presymptomatic, early symptomatic and late symptomatic stages for SCJD (MM1)-inoculated transgenic mice and at 120, 160 and 180 dpi for early symptomatic, symptomatic and late symptomatic to terminal stages respectively for sCJD (VV2)-inoculated transgenic mice. a Immunoflourescence experiment results showed an increase in DJ-1 expression and its oxidative stress-mediated nuclear translocalization in

phenylalanine-tRNA ligase beta subunit and elongation factor 1-alpha [45] could influence protein expression at translational level of protein synthesis. MicroRNA regulations in mice models of prion diseases [46, 47] are also well established. Identification of Staphylococcal nuclease domain-containing protein 1 (SND1) which regulates the activity of RISC
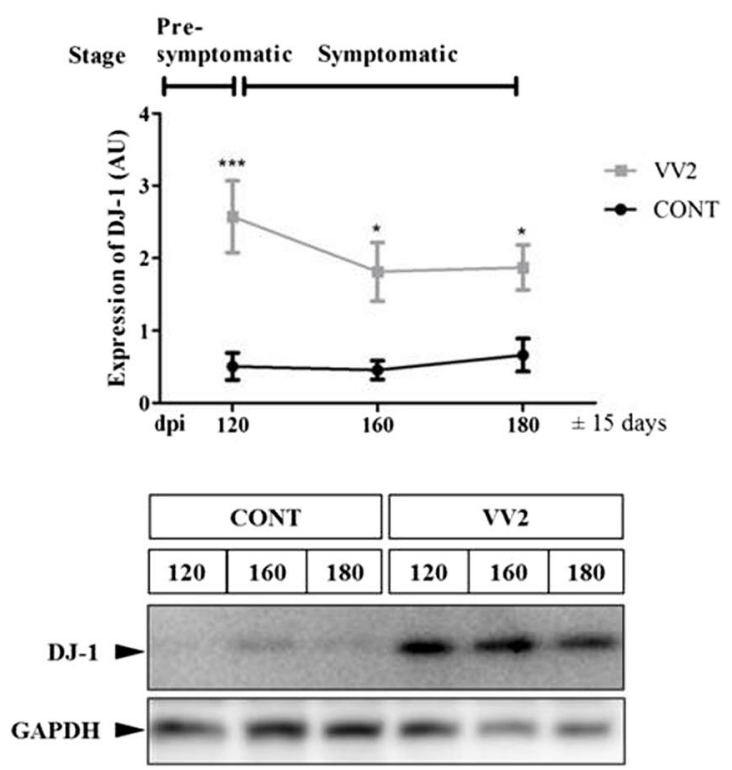

granular cell layer of cerebellum of SCJD (MM1)-inoculated transgenic mice at 183 dpi as compared to age-matched controls using anti DJ-1 primary antibody which confirms the expressional alteration of DJ-1 due to oxidative stress in SCJD (MM1)-inoculated transgenic mice as well. b DJ-1 expression in cerebellum of SCJD-MM1 transgenic mice with controls $(n=3)$ and MM1 $(n=3)$ for each time point and sCJD-VV2 transgenic mice with controls $(n=4)$ and VV2 $(n=4)$ for each time point was observed with western blot using DJ-1 antibody and densitometry analysis from three (for SCJD-MM1 transgenic mice) and from four (for sCJD-VV2 transgenic mice) independent $( \pm \mathrm{SD})$ western blotting experiments is shown. GAPDH was used as a loading control. The statistical significance was calculated with two-way ANOVA. Data are represented as the mean SEM. $* p<0.05, * * p<0.005, * * * p<0.001$

complex [48] could be responsible for the regulation of miRNA expressions in SCJD hence altering the protein expression at post-translational level. Identification of alterations of the abovementioned proteins strengthened the idea of the activation of gene expression regulatory cellular activities at different levels which could also be the reason for the 
unchanged mRNA expressions for $L D H B$, SUCLA-2 and $T D P$ (VV2) and increased mRNA expression for $P K M-2$ (in VV2) and TDP (in MM1).

Cellular stress response/oxidative stress-related functions were the second most regulated molecular functions in both subtypes (22\% in MM1 and 25\% in VV2) and included mainly dihydrolipoyl dehydrogenase which has a role in ROS production [49], AD and Parkinson's disease (PD) [50, 51]. Moreover, variations in the DLD gene have been linked to $\mathrm{AD}[52,53]$. Glutamine synthetase detoxifies brain ammonia in astrocytes and is increased in the CSF of AD patients [54] and is implicated in the pathogenesis of PD as well [55]. $\mathrm{N}(\mathrm{G})$-dimethylarginine dimethylaminohydrolase 1 modulates NO production and cell proliferation [56] and reported to be increased in the CSF of SCJD as well as in AD patients [57-59]; isocitrate dehydrogenase [NAD] subunit alpha (IDH1) produces NADPH during OS [60]; protein NDRG4 has an implication in cognition [61] and protection against neuronal cell death implied by ischemic stress [32], and superoxide dismutase 1 protects the cells against ROS. All of these proteins were identified to be altered in MM1 subtype. $\mathrm{X}$-ray repair cross-complementing protein 6 repairs the damaged DNA caused by ROS [62], and its genetic variations have been associated with the development of OS and PD [63]; heat shock protein $75 \mathrm{kDa}$ stabilizes the mitochondrial function under stress conditions, and electron transfer flavoprotein subunit alpha is responsible for mitochondrial energy metabolism under stress and is reported to be decreased in the brains of PINK1 (KO) mouse brains [64], were identified to be altered in VV2 subtype. Whereas dehydrogenase/reductase SDR family member 11 and glutathione S-transferase Mu 2 detoxify ROS are also implicated in PD [65], persulphide dioxygenase ETHE1 which catalyses the oxidation of $\mathrm{H}_{2} \mathrm{~S}$ in mitochondria [66,67], a reactive sulphur specie which is toxic for the cell; dihydropteridine reductase implicates its reducing nature to perform oxidoreductive functions; heat shock protein beta-1 prevents the misfolding of proteins in response to oxidative stress [68]; peroxiredoxin-6, thioredoxin-dependent peroxide reductase and protein DJ-1 are antioxidative in their function with later having a role in the early onset of familial form of PD [69], [70], [71] that were also identified to be altered in VV2 subtype.

Signal transduction and synaptic functions were the third most altered molecular functions in both subtypes (15\% in MM1 and 18\% in VV2) in this study and mainly comprised of complement $\mathrm{C} 3$; a member of complement system has a role in the initial trapping of prions in lymphoreticular organs immediately after infection [72] as well as in the exacerbation of disease pathology of chronic wasting disease in mouse [29]; protein kinase C delta-binding protein is implicated in the quick progression of motor symptoms in scrapie mouse [73]; guanine nucleotide-binding protein $\mathrm{G}(\mathrm{o})$ subunit alpha has a role in altered exocytosis in cerebral cortex of sCJD patients [74] [4]; phosphatase methylesterase 1 is implicated in $\mathrm{A} \beta$ production and tau phosphorylation; precipitating AD pathology [30] and syntaxin binding protein 1 which is implicated in fusion of synaptic vesicles [75] were identified commonly in both subtypes (MM1 and VV2). Hypoxanthine-guanine phosphoribosyl transferase and voltage-dependent anion-selective channel protein 2 have been implicated in PINK1 (KO) mouse brain [76], whereas transforming protein RhoA is reported to have a role in polymerization of actin filaments in SCJD patients [19], and phosphatidylinositol transfer protein alpha has a role in transportation of phosphoinositoles in different intracellular compartments for both trafficking and signalling purpose [77] identified to be altered in VV2 subtype.

Proteins having highest fold changes, peptide counts and sequence coverages in proteomic data set belonged mainly to either metabolic pathways or oxidative stress-related functions. Oxidative stress-related cellular activities were one of the major altered cellular activities based on proteomic findings in both subtypes (MM1 and VV2). Oxidative stress is one of the major contributors in various neurodegenerative diseases, but it is less previledged during the pathophysiology of the SCJD so far.

\section{Implication of Oxidative Stress in SCJD (as One of the Major Regulated Cellular Mechanisms)}

Oxidative stress refers to an imbalance of the equilibrium between the levels of ROS and cellular potential to detoxify these reactive intermediates, leading to perilous condition contributing to cellular and molecular damage [78]. This study revealed the presence of reactive sulphur species along with ROS and RNS which could lead to multiple toxic mechanisms in the brain. OS in a cell can deregulate many of cellular functions like deregulation of metabolic pathways [79], impairment of energy-producing machinery of cell [80], signal transduction [81], activation of apoptotic pathways [82] and misfolding of proteins as well as aggregation of proteins [83, 84]. Elevated oxidation, glycoxidation, lipoxidation and nitrative protein damage only in cerebral cortex in sporadic CJD and prion-inoculated syrian hamster have been reported $[85,86]$ as well. So, OS could be assumed as one of the important contributors to the rapid progression of disease pathology in SCJD.

\section{Role of DJ-1 in sCJD During OS}

Protein deglycase (DJ-1) was one of the proteins found altered in this proteomic data set. DJ-1 was found significantly increased at mRNA level in both MM1 and VV2 subtypes, whereas only in VV2 subtype at protein level. DJ-1 is known as a sensor of OS and for its role in 
protection against OS. Mutations in the DJ-1 gene have been associated with autosomal recessive familial PD [80]. DJ-1 protects the cells against OS by self-oxidation to quench ROS [17, 53, 64], and excessively oxidized DJ1 has been reported in terminal brains of PD and AD patients [34]. At the same time, DJ-1 is not found in Lewy bodies [87] but shows co-localization with Tau in neurofibrillary tangles in brains of AD patients, suggesting the role of DJ-1 in AD as well [63, 74]. Baulac et al. have reported increased levels of DJ-1 in AD brains as well [71]. This is the first time that DJ-1 has been identified to be altered in the brain of SCJD patients. Its conserved sequence throughout the evolution implicates its neuroprotective function by regulating antioxidative, antiapoptotic and antiinflammatory pathways [88]. During oxidative stress, DJ-1 acts as an ROS quencher, resulting in translocalization to mitochondria as well as nucleus from the cytoplasm [89, 90]. Oxidation of DJ-1 at Cys 106 residue to sulfinic acid is critical for DJ-1 to play its neuroprotective role by interacting with other proteins [91]. In this study, nuclear translocation of DJ-1 strengthened the idea of its expressional alteration due to OS in cerebellum of SCJD (MM1) human as well as CJD (MM1) mice. Furthermore, higher expression of DJ-1 at presymptomatic stage of CJD in mice models of SCJD indicates OS as one of the earliest disease events in sCJD. OS can be both a cause of neurodegeneration [83, 89] and protein misfolding [78] and also an effect during the pathophysiology of sCJD [92]. In this study, increased expression of DJ-1 indicated higher levels of OS at presymptomatic stage of disease. But this cellular response seemed to be collapsing during the disease progression. This effect could be due to the lack of cellular energy [31] or mitochondrial damage [92] leading to no further increase in the expression of DJ-1 at symptomatic stage of the disease in comparison to presymptomatic stage of CJD in mice.

\section{Direct Role of DJ-1 in Protection Against OS by Mediating Thioredoxin System}

Thioredoxin-dependent peroxide reductase was found significantly increased in VV2 subtype based on our proteomic findings. It is an important component in the thioredoxin system. Thioredoxin (Trx) system is a small family of antioxidant enzymes involved in neuroprotection by alleviating oxidative stress $[87,93,94]$. Trx also protects the cells by regulating the activity of apoptosis signal-regulating kinase-1 (ASK1) [33]. Wild-type DJ-1 can inhibit the apoptotic potential of AKS1 by interacting with it and blocking its dissociation from Trx 1 [95]. Collectively, DJ-1-ASK1 interaction plays a vital role in the antioxidant response by modulating the interaction between ASK1 and Trx1.

\section{Direct Role of DJ-1 in Protection Against OS by Mediating Glutathione Biosynthesis}

Glutathione (GSH) is another antioxidant protecting the cells from free radicals, peroxides, lipid peroxides and heavy metals [96]. DJ-1 also regulates the glutathione activity by increasing the expression of the enzyme GCL which is the first enzyme of the pathway of cellular GSH biosynthesis [97]. Glutathione S-transferase Mu 2 is a class of enzymes which protects the cells by detoxifying electrophilic compounds like carcinogens, drug residues, environmental toxins and toxic products of oxidative stress [98]. GST Mu 2 expression was found significantly increased both at transcriptional and translational level in MM1 and VV2 subtypes of SCJD in present study.

\section{Indirect Role of DJ-1 in Protection Against OS by Mediating Nrf2/ARE Pathway}

One of the proposed mechanism of action of protecting the cell against OS by DJ-1 is through regulating the activity of

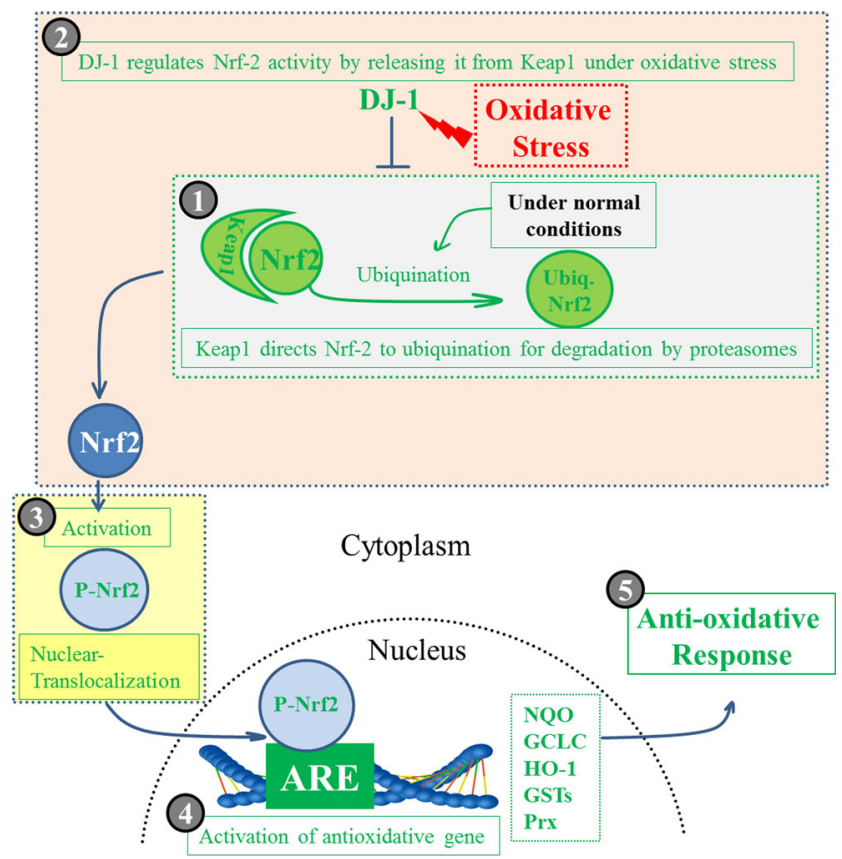

Fig. 7 Mechanism of antioxidative response activation of DJ-1 by mediating Nrf2/ARE pathway: One of the mechanisms by which DJ-1 protects the cells against oxidative stress is by regulating the activity of Nrf2 which leads to activation of antioxidative response elements (AREs) in the nucleus. Under normal cellular conditions, Nrf2 activity is inhibited by Keap 1 who continuously ubiquinates Nrf2 and directs to proteasomal degradation (1). Under the effect of oxidative stress, DJ-1 protein expression is increased and it regulates $\mathrm{Nrf} 2$ activity and releases $\mathrm{Nrf} 2$ from Keap1 for its downstream functions (2). Nrf2 is phosphorylated which is necessary for its downstream activity, and then, it translocalizes to the nucleus (3). In the nucleus, phospho-Nrf2 induces the expression of antioxidative genes NQO1, HO-1, GSLs, GSTs and Prx (4). Expression of these antioxidative genes then performs their functions in a variety of ways to protect the cell against OS (5) 
the transcription factor Nrf2. Nrf2 induces the expression of ARE and is reported to be regulated by DJ-1 [99]. Increased expression of DJ-1 in response to OS leads to inactivation of Keap1 resulting in the release of Nrf2 from Keap1, inducing its translocation to the nucleus and thus activating ARE which is the main regulatory site of antioxidant expression [28, 100]. Keap1 is the main regulator of Nrf2 activity by continuously ubiquitinating $\mathrm{Nrf} 2$ in cytoplasm and directing it to proteasomal degradation. But during oxidative stress, Keap1 is inactivated and Nrf2 expression is stabilized by DJ-1 [99]. Our results showing increased mRNA expression of Nrf2, decreased expression of ubiquinated-Nrf2 in both MM1 and VV2 subtypes, along with high expression of its activated form, phospho-Nrf2 predominately in VV2 subtype confirmed the activation of Nrf2 in SCJD (MM1 and VV2 subtype) cases for downstream regulation of ARE. ARE encode detoxifying enzymes and antioxidant proteins, including NAD(P)H:quinone oxidoreductase 1, hemeoxygenase-1, glutathione S-transferase, glutathione cysteine ligase $\mathrm{C}$, glutathione peroxidase and thioredoxin-dependent peroxide reductase.

Alteration in the expression of hemeoxygenase-1, thioredoxin-dependent peroxide reductase and glutathione Stransferase $\mathrm{Mu} 2$ confirmed the activation of complete Nrf2/ARE pathway in sCJD (MM1 and VV2 subtypes) (Fig. 7). Target genes of Nrf2 are generally involved in glutathione synthesis, elimination of ROS, xenobiotic metabolism and drug transport [92, 101-103].

\section{Conclusion}

Based on these results, we speculate that oxidative stress is one of the major pathological factors during the course of the disease in MM1 and VV2 subtypes of SCJD which can deregulate other cellular activities as well. Furthermore, oxidative stress is present even during the presymptomatic stage of the disease in SCJD as shown by results from SCJD (MM1 and VV2)-inoculated transgenic mice. DJ-1 is commonly playing its antioxidative role (both directly by localizing to the nucleus and indirectly by activating Nrf2/ARE pathway) in SCJD. Using antioxidative therapeutic strategies in the future may be helpful not only in decreasing the progression rate of SCJD specifically but also at some point, it may be helpful to overcome the pronounced levels of oxidative stress and to recover the cell from pathological condition. Furthermore, these results provide a broader overview of the whole regulated proteome during the course of disease in SCJD (MM1 and VV2 subtypes) in human, thus opening the new horizons for better understanding the pathophysiology of disease and potentially redirecting the therapeutic strategies.

\section{Supporting Information}

Supporting information is provided in separate files. Supporting information file 1 contains raw files of the all the identified proteins received after mass spectrometric identification. Supporting information file 2 contains MS data including MS/MS spectra for all proteins identified in MS excel format. Supporting information file 3 contains discussion on the functions of all the identified regulated proteins with details.

Acknowledgements This work was supported by a grant from Helmholtz-Alberta Initiative-Infectious Diseases Research (HAI-IDR) and APRI-Human prions - distinguishing sporadic from familial forms via structure and function as well as from the DZNE clinical project (Helmholtz). The study was performed within the recently established Clinical Dementia Center at the University Medical Hospital Göttingen and was partly supported by grants from the EU Joint Program Neurodegenerative Disease Research (JPND-DEMTEST) (Biomarker based diagnosis of rapid progressive dementias-optimization of diagnostic protocols, 01ED1201A). We also pay our special thanks to Dr. André Fischer (DZNE, Goettingen) and Dr. Stefan Bonn (DZNE, Goettingen) for sharing their lab facilities, Dr. Christine Stadelmann-Nessler (UMG, Goettingen) for providing hemeoxygenase 1 primary antibody and Dr. Hassan Dihazi (UMG, Goettingen) for peroxiredoxin 6 primary antibody.

\section{References}

1. Prusiner SB, DeArmond SJ (1994) Prion diseases and neurodegeneration. Annu Rev Neurosci 17:311-339

2. Gambetti P, Kong Q, Zou W, Parchi P, Chen SG (2003) Sporadic and familial CJD: classification and characterisation. Br Med Bull 66:213-239

3. Puoti G, Bizzi A, Forloni G, Safar JG, Tagliavini F, Gambetti P (2012) Sporadic human prion diseases: molecular insights and diagnosis. Lancet Neurol 11(7):618-628

4. Gawinecka J, Cardone F, Asif AR, De PA, Wemheuer WM, Schulz-Schaeffer WJ, Pocchiari M, Zerr I (2012) Sporadic creutzfeldt-Jakob disease subtype-specific alterations of the brain proteome: impact on Rab3a recycling. Proteomics 12(23-24): $3610-3620$

5. Parchi P, Giese A, Capellari S, Brown P, Schulz-Schaeffer W, Windl O, Zerr I, Budka H et al (1999) Classification of sporadic Creutzfeldt-Jakob disease based on molecular and phenotypic analysis of 300 subjects. Ann Neurol 46(2):224-233

6. Yang Q, Hashizume Y, Yoshida M, Wang Y (1999) Neuropathological study of cerebellar degeneration in prion disease. Neuropathology 19(1):33-39

7. KH E, Chen SH, Ho MH, Desmond JE (2014) A meta-analysis of cerebellar contributions to higher cognition from PET and fMRI studies. Hum Brain Mapp 35(2):593-615

8. Kim SG, Ugurbil K, Strick PL (1994) Activation of a cerebellar output nucleus during cognitive processing. Science 265(5174): 949-951

9. Zhang J, Keene CD, Pan C, Montine KS, Montine TJ (2008) Proteomics of human neurodegenerative diseases. J Neuropathol Exp Neurol 67(10):923-932

10. Yun SW, Gerlach M, Riederer P, Klein MA (2006) Oxidative stress in the brain at early preclinical stages of mouse scrapie. Exp Neurol 201(1):90-98 
11. Harish G, Venkateshappa C, Mahadevan A, Pruthi N, Bharath MM, Shankar SK (2011) Effect of storage time, postmortem interval, agonal state, and gender on the postmortem preservation of glial fibrillary acidic protein and oxidatively damaged proteins in human brains. Biopreserv Biobank 9(4):379-387

12. Llorens F, Ansoleaga B, Garcia-Esparcia P, Zafar S, Grau-Rivera O, Lopez-Gonzalez I, Blanco R, Carmona M et al (2013) PrP mRNA and protein expression in brain and $\operatorname{PrP}(\mathrm{c})$ in $\mathrm{CSF}$ in Creutzfeldt-Jakob disease MM1 and VV2. Prion 7(5):383-393

13. Llorens F, Lopez-Gonzalez I, Thune K, Carmona M, Zafar S, Andreoletti O, Zerr I, Ferrer I (2014) Subtype and regionalspecific neuroinflammation in sporadic creutzfeldt-jakob disease. Front Aging Neurosci 6:198

14. Zafar S, Asif AR, Ramljak S, Tahir W, Schmitz M, Zerr I (2014) Anchorless 23-230 PrPC interactomics for elucidation of PrPC protective role. Mol Neurobiol 49(3):1385-1399

15. Ramljak S, Asif AR, Armstrong VW, Wrede A, Groschup MH, Buschmann A, Schulz-Schaeffer W, Bodemer W et al (2008) Physiological role of the cellular prion protein $(\mathrm{PrPc})$ : protein profiling study in two cell culture systems. J Proteome Res 7(7): 2681-2695

16. Blum H, Beier H, Gross HJ (1987) Improved silver staining of plant proteins, RNA and DNA in polyacrylamide gels. Electrophoresis 8(2):93-99

17. Cassard H, Torres JM, Lacroux C, Douet JY, Benestad SL, Lantier F, Lugan S, Lantier I et al (2014) Evidence for zoonotic potential of ovine scrapie prions. Nat Commun 5:5821

18. Padilla D, Beringue V, Espinosa JC, Andreoletti O, Jaumain E, Reine F, Herzog L, Gutierrez-Adan A et al (2011) Sheep and goat BSE propagate more efficiently than cattle BSE in human PrP transgenic mice. PLoS Pathog 7(3):e1001319

19. Zafar S, Schmitz M, Younus N, Tahir W, Shafiq M, Llorens F, Ferrer I, Andeoletti O et al (2015) Creutzfeldt-Jakob disease subtype-specific regional and temporal regulation of ADP Ribosylation factor-1-dependent rho/MLC pathway at preclinical stage. J Mol Neurosci 56(2):329-348

20. Zafar S, Younas N, Correia S, Shafiq M, Tahir W, Schmitz M, Ferrer I, Andreoletti O et al (2016) Strain-specific altered regulatory response of Rab7a and Tau in Creutzfeldt-Jakob disease and Alzheimer's disease. Mol Neurobiol. doi:10.1007/s12035-0169694-8

21. Kruse N, Schulz-Schaeffer WJ, Schlossmacher MG, Mollenhauer B (2012) Development of electrochemiluminescence-based singleplex and multiplex assays for the quantification of alphasynuclein and other proteins in cerebrospinal fluid. Methods 56(4):514-518

22. Blackinton J, Lakshminarasimhan M, Thomas KJ, Ahmad R, Greggio E, Raza AS, Cookson MR, Wilson MA (2009) Formation of a stabilized cysteine sulfinic acid is critical for the mitochondrial function of the parkinsonism protein DJ-1. J Biol Chem 284(10):6476-6485

23. Kim SJ, Park YJ, Hwang IY, Youdim MB, Park KS, Oh YJ (2012) Nuclear translocation of DJ-1 during oxidative stress-induced neuronal cell death. Free Radic Biol Med 53(4):936-950

24. Liu C, Chen Y, Kochevar IE, Jurkunas UV (2014) Decreased DJ-1 leads to impaired Nrf2-regulated antioxidant defense and increased UV-A-induced apoptosis in corneal endothelial cells. Invest Ophthalmol Vis Sci 55(9):5551-5560

25. Itoh $\mathrm{K}$, Chiba $\mathrm{T}$, Takahashi $\mathrm{S}$, Ishii $\mathrm{T}$, Igarashi $\mathrm{K}$, Katoh $\mathrm{Y}$, Oyake T, Hayashi N et al (1997) An Nrf2/small Maf heterodimer mediates the induction of phase II detoxifying enzyme genes through antioxidant response elements. Biochem Biophys Res Commun 236(2):313-322

26. Venugopal R, Jaiswal AK (1996) Nrf1 and Nrf2 positively and cFos and Fra1 negatively regulate the human antioxidant response element-mediated expression of $\mathrm{NAD}(\mathrm{P}) \mathrm{H}$ :quinone oxidoreductasel gene. Proc Natl Acad Sci U S A 93(25):14960-14965

27. Ayala YM, De CL, Avendano-Vazquez SE, Dhir A, Romano M, D'Ambrogio A, Tollervey J, Ule J et al (2011) TDP-43 regulates its mRNA levels through a negative feedback loop. EMBO J 30(2):277-288

28. Malhotra D, Thimmulappa R, Navas-Acien A, Sandford A, Elliott M, Singh A, Chen L, Zhuang X et al (2008) Decline in NRF2regulated antioxidants in chronic obstructive pulmonary disease lungs due to loss of its positive regulator, DJ-1. Am J Respir Crit Care Med 178(6):592-604

29. Michel B, Ferguson A, Johnson T, Bender H, Meyerett-Reid C, Wyckoff AC, Pulford B, Telling GC et al (2013) Complement protein $\mathrm{C} 3$ exacerbates prion disease in a mouse model of chronic wasting disease. Int Immunol 25(12):697-702

30. Mokhtar SH, Bakhuraysah MM, Cram DS, Petratos S (2013) The beta-amyloid protein of Alzheimer's disease: communication breakdown by modifying the neuronal cytoskeleton. Int J Alzheimers Dis 2013:910502

31. Zhou M, Ottenberg G, Sferrazza GF, Hubbs C, Fallahi M, Rumbaugh G, Brantley AF, Lasmezas CI (2015) Neuronal death induced by misfolded prion protein is due to NAD+ depletion and can be relieved in vitro and in vivo by NAD+ replenishment. Brain 138(Pt 4):992-1008

32. Zhou RH, Kokame K, Tsukamoto Y, Yutani C, Kato H, Miyata T (2001) Characterization of the human NDRG gene family: a newly identified member, NDRG4, is specifically expressed in brain and heart. Genomics 73(1):86-97

33. Saitoh M, Nishitoh H, Fujii M, Takeda K, Tobiume K, Sawada Y, Kawabata M, Miyazono K et al (1998) Mammalian thioredoxin is a direct inhibitor of apoptosis signal-regulating kinase (ASK) 1 . EMBO J 17(9):2596-2606

34. Buratti E, Baralle FE (2010) The multiple roles of TDP-43 in premRNA processing and gene expression regulation. RNA Biol 7(4):420-429

35. Buratti E, Baralle M, Baralle FE (2013) From single splicing events to thousands: the ambiguous step forward in splicing research. Brief Funct Genomics 12(1):3-12

36. Buratti E, Romano M, Baralle FE (2013) TDP-43 high throughput screening analyses in neurodegeneration: advantages and pitfalls. Mol Cell Neurosci 56:465-474

37. Iguchi Y, Katsuno M, Niwa J, Takagi S, Ishigaki S, Ikenaka K, Kawai K, Watanabe H et al (2013) Loss of TDP-43 causes agedependent progressive motor neuron degeneration. Brain 136(Pt 5):1371-1382

38. Licatalosi DD, Darnell RB (2010) RNA processing and its regulation: global insights into biological networks. Nat Rev Genet 11(1):75-87

39. Vance C, Rogelj B, Hortobagyi T, De Vos KJ, Nishimura AL, Sreedharan J, Hu X, Smith B et al (2009) Mutations in FUS, an RNA processing protein, cause familial amyotrophic lateral sclerosis type 6 . Science 323(5918):1208-1211

40. Perrone-Bizzozero N, Bolognani F (2002) Role of HuD and other RNA-binding proteins in neural development and plasticity. $\mathrm{J}$ Neurosci Res 68(2):121-126

41. Bekenstein U, Soreq H (2013) Heterogeneous nuclear ribonucleoprotein A1 in health and neurodegenerative disease: from structural insights to post-transcriptional regulatory roles. Mol Cell Neurosci 56:436-446

42. Berson A, Barbash S, Shaltiel G, Goll Y, Hanin G, Greenberg DS, Ketzef M, Bcker AJ et al (2012) Cholinergic-associated loss of hnRNP-A/B in Alzheimer's disease impairs cortical splicing and cognitive function in mice. EMBO Mol Med 4(8):730-742

43. Park SJ, Lee H, Jo DS, Jo YK, Shin JH, Kim HB, Seo HM, Rubinsztein DC et al (2015) Heterogeneous nuclear 
ribonucleoprotein A1 post-transcriptionally regulates Drp1 expression in neuroblastoma cells. Biochim Biophys Acta 1849(12):1423-1431

44. Amadio M, Scapagnini G, Davinelli S, Calabrese V, Govoni S, Pascale A (2014) Involvement of ELAV RNA-binding proteins in the post-transcriptional regulation of HO-1. Front Cell Neurosci 8: 459

45. Becker M, Kuhse J, Kirsch J (2013) Effects of two elongation factor $1 \mathrm{~A}$ isoforms on the formation of gephyrin clusters at inhibitory synapses in hippocampal neurons. Histochem Cell Biol 140(6):603-609

46. Boese AS, Saba R, Campbell K, Majer A, Medina S, Burton L, Booth TF, Chong P et al (2016) MicroRNA abundance is altered in synaptoneurosomes during prion disease. Mol Cell Neurosci 71:13-24

47. Saba R, Goodman CD, Huzarewich RL, Robertson C, Booth SA (2008) A miRNA signature of prion induced neurodegeneration. PLoS One 3(11):e3652

48. Tsuchiya N, Ochiai M, Nakashima K, Ubagai T, Sugimura T, Nakagama H (2007) SND1, a component of RNA-induced silencing complex, is up-regulated in human colon cancers and implicated in early stage colon carcinogenesis. Cancer Res 67(19): 9568-9576

49. Tahara EB, Barros MH, Oliveira GA, Netto LE, Kowaltowski AJ (2007) Dihydrolipoyl dehydrogenase as a source of reactive oxygen species inhibited by caloric restriction and involved in Saccharomyces cerevisiae aging. FASEB J 21(1):274-283

50. Gibson GE, Park LC, Sheu KF, Blass JP, Calingasan NY (2000) The alpha-ketoglutarate dehydrogenase complex in neurodegeneration. Neurochem Int 36(2):97-112

51. Sullivan PG, Brown MR (2005) Mitochondrial aging and dysfunction in Alzheimer's disease. Prog Neuro-Psychopharmacol Biol Psychiatry 29(3):407-410

52. Brown AM, Gordon D, Lee H, Caudy M, Haroutunian V, Blass JP (2004) Substantial linkage disequilibrium across the dihydrolipoyl succinyltransferase gene region without Alzheimer's disease association. Neurochem Res 29(3):629-635

53. Brown AM, Gordon D, Lee H, Wavrant-De VF, Cellini E, Bagnoli S, Nacmias B, Sorbi S et al (2007) Testing for linkage and association across the dihydrolipoyl dehydrogenase gene region with Alzheimer's disease in three sample populations. Neurochem Res 32(4-5):857-869

54. Tumani H, Shen G, Peter JB, Bruck W (1999) Glutamine synthetase in cerebrospinal fluid, serum, and brain: a diagnostic marker for Alzheimer disease? Arch Neurol 56(10):1241-1246

55. Plaitakis A, Shashidharan P (2000) Glutamate transport and metabolism in dopaminergic neurons of substantia nigra: implications for the pathogenesis of Parkinson's disease. J Neurol 247(Suppl 2):II25-II35

56. Zhang P, Hu X, Xu X, Chen Y, Bache RJ (2011) Dimethylarginine dimethylaminohydrolase 1 modulates endothelial cell growth through nitric oxide and Akt. Arterioscler Thromb Vasc Biol 31(4):890-897

57. Butterfield DA, Gnjec A, Poon HF, Castegna A, Pierce WM, Klein JB, Martins RN (2006) Redox proteomics identification of oxidatively modified brain proteins in inherited Alzheimer's disease: an initial assessment. J Alzheimers Dis 10(4):391-397

58. Gawinecka J, Dieks J, Asif AR, Carimalo J, Heinemann U, Streich JH, Dihazi H, Schulz-Schaeffer W et al (2010) Codon 129 polymorphism specific cerebrospinal fluid proteome pattern in sporadic Creutzfeldt-Jakob disease and the implication of glycolytic enzymes in prion-induced pathology. J Proteome Res 9(11):56465657

59. Mulder C, Wahlund LO, Blomberg M, de JS, van Kamp GJ, Scheltens P, Teerlink T (2002) Alzheimer's disease is not associated with altered concentrations of the nitric oxide synthase inhibitor asymmetric dimethylarginine in cerebrospinal fluid. $\mathrm{J}$ Neural Transm (Vienna) 109(9):1203-1208

60. Molenaar RJ, Radivoyevitch T, Maciejewski JP, van Noorden CJ, Bleeker FE (2014) The driver and passenger effects of isocitrate dehydrogenase 1 and 2 mutations in oncogenesis and survival prolongation. Biochim Biophys Acta 1846(2):326-341

61. Yamamoto H, Kokame K, Okuda T, Nakajo Y, Yanamoto H, Miyata T (2011) NDRG4 protein-deficient mice exhibit spatial learning deficits and vulnerabilities to cerebral ischemia. J Biol Chem 286(29):26158-26165

62. Vidal AE, Boiteux S, Hickson ID, Radicella JP (2001) XRCC1 coordinates the initial and late stages of DNA abasic site repair through protein-protein interactions. EMBO J 20(22):6530-6539

63. Gencer M, Dasdemir S, Cakmakoglu B, Cetinkaya Y, Varlibas F, Tireli H, Kucukali CI, Ozkok E et al (2012) DNA repair genes in Parkinson's disease. Genet Test Mol Biomarkers 16(6):504-507

64. Diedrich M, Kitada T, Nebrich G, Koppelstaetter A, Shen J, Zabel C, Klose J, Mao L (2011) Brain region specific mitophagy capacity could contribute to selective neuronal vulnerability in Parkinson's disease. Proteome Sci 9:59

65. Smeyne M, Smeyne RJ (2013) Glutathione metabolism and Parkinson's disease. Free Radic Biol Med 62:13-25

66. Kabil O, Banerjee R (2012) Characterization of patient mutations in human persulfide dioxygenase (ETHE1) involved in $\mathrm{H} 2 \mathrm{~S}$ catabolism. J Biol Chem 287(53):44561-44567

67. Pettinati I, Brem J, McDonough MA, Schofield CJ (2015) Crystal structure of human persulfide dioxygenase: structural basis of ethylmalonic encephalopathy. Hum Mol Genet 24(9):2458-2469

68. Di DF, Sultana R, Tiu GF, Scheff NN, Perluigi M, Cini C, Butterfield DA (2010) Protein levels of heat shock proteins 27, $32,60,70,90$ and thioredoxin-1 in amnestic mild cognitive impairment: an investigation on the role of cellular stress response in the progression of Alzheimer disease. Brain Res 1333:72-81

69. Power JH, Asad S, Chataway TK, Chegini F, Manavis J, Temlett JA, Jensen PH, Blumbergs PC et al (2008) Peroxiredoxin 6 in human brain: molecular forms, cellular distribution and association with Alzheimer's disease pathology. Acta Neuropathol 115(6):611-622

70. Silva-Adaya D, Gonsebatt ME, Guevara J (2014) Thioredoxin system regulation in the central nervous system: experimental models and clinical evidence. Oxidative Med Cell Longev 2014: 590808

71. Baulac S, Lu H, Strahle J, Yang T, Goldberg MS, Shen J, Schlossmacher MG, Lemere CA et al (2009) Increased DJ-1 expression under oxidative stress and in Alzheimer's disease brains. Mol Neurodegener 4:12

72. Klein MA, Kaeser PS, Schwarz P, Weyd H, Xenarios I, Zinkernagel RM, Carroll MC, Verbeek JS et al (2001) Complement facilitates early prion pathogenesis. Nat Med 7(4): 488-492

73. Harischandra DS, Kondru N, Martin DP, Kanthasamy A, Jin H, Anantharam V, Kanthasamy AG (2014) Role of proteolytic activation of protein kinase Cdelta in the pathogenesis of prion disease. Prion 8(1):143-153

74. Eaton CJ, Cabrera IE, Servin JA, Wright SJ, Cox MP, Borkovich KA (2012) The guanine nucleotide exchange factor RIC8 regulates conidial germination through Galpha proteins in Neurospora crassa. PLoS One 7(10):e48026

75. Rizo J, Sudhof TC (2002) Snares and Munc18 in synaptic vesicle fusion. Nat Rev Neurosci 3(8):641-653

76. Triplett JC, Zhang Z, Sultana R, Cai J, Klein JB, Bueler H, Butterfield DA (2015) Quantitative expression proteomics and phosphoproteomics profile of brain from PINK1 knockout mice: insights into mechanisms of familial Parkinson's disease. J Neurochem 133(5):750-765 
77. Hsuan J, Cockcroft S 2001 The PITP family of phosphatidylinositol transfer proteins. Genome Biol 2(9):REVIEWS3011

78. Betteridge DJ (2000) What is oxidative stress? Metabolism 49(2 Suppl 1):3-8

79. Dringen R, Gutterer JM, Hirrlinger J (2000) Glutathione metabolism in brain metabolic interaction between astrocytes and neurons in the defense against reactive oxygen species. Eur J Biochem 267(16):4912-4916

80. Gandhi S, Abramov AY (2012) Mechanism of oxidative stress in neurodegeneration. Oxidative Med Cell Longev 2012:428010

81. Apel K, Hirt H (2004) Reactive oxygen species: metabolism, oxidative stress, and signal transduction. Annu Rev Plant Biol 55: 373-399

82. Salganik RI (2001) The benefits and hazards of antioxidants: controlling apoptosis and other protective mechanisms in cancer patients and the human population. J Am Coll Nutr 20(5 Suppl): 464S-472S

83. Bruijn LI, Houseweart MK, Kato S, Anderson KL, Anderson SD, Ohama E, Reaume AG, Scott RW et al (1998) Aggregation and motor neuron toxicity of an ALS-linked SOD1 mutant independent from wild-type SOD1. Science 281(5384):1851-1854

84. Valentine JS, Hart PJ (2003) Misfolded CuZnSOD and amyotrophic lateral sclerosis. Proc Natl Acad Sci U S A 100(7):3617-3622

85. Freixes M, Rodriguez A, Dalfo E, Ferrer I (2006) Oxidation, glycoxidation, lipoxidation, nitration, and responses to oxidative stress in the cerebral cortex in creutzfeldt-Jakob disease. Neurobiol Aging 27(12):1807-1815

86. Pamplona R, Naudi A, Gavin R, Pastrana MA, Sajnani G, Ilieva EV, Del Rio JA, Portero-Otin M et al (2008) Increased oxidation, glycoxidation, and lipoxidation of brain proteins in prion disease. Free Radic Biol Med 45(8):1159-1166

87. Bai J, Nakamura H, Hattori I, Tanito M, Yodoi J (2002) Thioredoxin suppresses 1-methyl-4-phenylpyridinium-induced neurotoxicity in rat PC12 cells. Neurosci Lett 321(1-2):81-84

88. Kahle PJ, Waak J, Gasser T (2009) DJ-1 and prevention of oxidative stress in Parkinson's disease and other age-related disorders. Free Radic Biol Med 47(10):1354-1361

89. Canet-Aviles RM, Wilson MA, Miller DW, Ahmad R, McLendon C, Bandyopadhyay S, Baptista MJ, Ringe D et al (2004) The Parkinson's disease protein DJ-1 is neuroprotective due to cysteine-sulfinic acid-driven mitochondrial localization. Proc Natl Acad Sci U S A 101(24):9103-9108

90. Junn E, Jang WH, Zhao X, Jeong BS, Mouradian MM (2009) Mitochondrial localization of DJ-1 leads to enhanced neuroprotection. J Neurosci Res 87(1):123-129

91. Kato I, Maita H, Takahashi-Niki K, Saito Y, Noguchi N, IguchiAriga SM, Ariga H (2013) Oxidized DJ-1 inhibits p53 by sequestering p53 from promoters in a DNA-binding affinity-dependent manner. Mol Cell Biol 33(2):340-359
92. Chanas SA, Jiang Q, McMahon M, McWalter GK, McLellan LI, Elcombe CR, Henderson CJ, Wolf CR et al (2002) Loss of the $\mathrm{Nrf} 2$ transcription factor causes a marked reduction in constitutive and inducible expression of the glutathione S-transferase Gstal, Gsta2, Gstm1, Gstm2, Gstm3 and Gstm4 genes in the livers of male and female mice. Biochem J 365(Pt 2):405-416

93. Takagi Y, Hattori I, Nozaki K, Mitsui A, Ishikawa M, Hashimoto N, Yodoi J (2000) Excitotoxic hippocampal injury is attenuated in thioredoxin transgenic mice. J Cereb Blood Flow Metab 20(5): 829-833

94. Zhou F, Gomi M, Fujimoto M, Hayase M, Marumo T, Masutani $\mathrm{H}$, Yodoi J, Hashimoto N et al (2009) Attenuation of neuronal degeneration in thioredoxin-1 overexpressing mice after mild focal ischemia. Brain Res 1272:62-70

95. Im JY, Lee KW, Junn E, Mouradian MM (2010) DJ-1 protects against oxidative damage by regulating the thioredoxin/ASK1 complex. Neurosci Res 67(3):203-208

96. Pompella A, Visvikis A, Paolicchi A, De TV, Casini AF (2003) The changing faces of glutathione, a cellular protagonist. Biochem Pharmacol 66(8):1499-1503

97. Zhou W, Freed CR (2005) DJ-1 up-regulates glutathione synthesis during oxidative stress and inhibits A53T alpha-synuclein toxicity. J Biol Chem 280(52):43150-43158

98. Li Y, Yan M, Yang J, Raman I, Du Y, Min S, Fang X, Mohan C et al (2014) Glutathione S-transferase mu 2-transduced mesenchymal stem cells ameliorated anti-glomerular basement membrane antibody-induced glomerulonephritis by inhibiting oxidation and inflammation. Stem Cell Res Ther 5(1):19

99. Clements CM, McNally RS, Conti BJ, Mak TW, Ting JP (2006) DJ-1, a cancer- and Parkinson's disease-associated protein, stabilizes the antioxidant transcriptional master regulator Nrf2. Proc Natl Acad Sci U S A 103(41):15091-15096

100. Cheng X, Chapple SJ, Patel B, Puszyk W, Sugden D, Yin X, Mayr M, Siow RC et al (2013) Gestational diabetes mellitus impairs Nrf2-mediated adaptive antioxidant defenses and redox signaling in fetal endothelial cells in utero. Diabetes 62(12):4088-4097

101. Moinova HR, Mulcahy RT (1999) Up-regulation of the human gamma-glutamylcysteine synthetase regulatory subunit gene involves binding of $\mathrm{Nrf}-2$ to an electrophile responsive element. Biochem Biophys Res Commun 261(3):661-668

102. Sekhar KR, Crooks PA, Sonar VN, Friedman DB, Chan JY, Meredith MJ, Starnes JH, Kelton KR et al (2003) NADPH oxidase activity is essential for Keap1/Nrf2-mediated induction of GCLC in response to 2-indol-3-yl-methylenequinuclidin-3-ols. Cancer Res 63(17):5636-5645

103. Thimmulappa RK, Mai KH, Srisuma S, Kensler TW, Yamamoto M, Biswal S (2002) Identification of Nrf2-regulated genes induced by the chemopreventive agent sulforaphane by oligonucleotide microarray. Cancer Res 62(18):5196-5203 